\title{
Das Prinzip der Gerechtigkeit im Steuerwesen nach dem Zweiten Weltkrieg
}

In der Zeit von 1945 bis 1948/1949 ging der Wiederaufbau der Finanzen in den vier Besatzungszonen unterschiedliche Wege. Von einer zielgerichteten Finanzpolitik konnte man nicht sprechen, doch blieb die Einheit der Besteuerung durch den Alliierten Kontrollrat gewahrt. Der Alliierte Kontrollrat erhöhte die Tarife der Einkommen-, Körperschaft-, Umsatz- und Vermögensteuer sowie die Verbrauchsteuern drastisch. Der Spitzeneinkommensteuersatz wurde auf 94 Prozent, die Vermögensteuer auf 2,5 Prozent und die Umsatzsteuer auf 3 Prozent angehoben. Die Zielsetzung dieser hohen Besteuerung bestand darin, den inflationären Geldüberhang abzubauen, gleichzeitig war dies aber ein wesentliches Hindernis für eine wirtschaftliche Entwicklung. ${ }^{983}$

Vier Jahre nach dem Ende des Zweiten Weltkrieges gingen aus den vier Besatzungszonen zwei Staaten mit zwei verschiedenen Finanzsystemen hervor. In der DDR entstand eine Zentralplanwirtschaft. Ein Großteil der Produktionsmittel war Staatseigentum, die Wirtschaft wurde durch Mehrjahrespläne zentral gelenkt. Haupteinnahmequelle des Haushalts waren die Abgaben der Volkeigenen Betriebe

983 Ehrlicher, Werner: Finanzpolitik seit 1945, in: Schremmer, Eckart (Hrsg.): Steuern, Abgaben und Dienste vom Mittelalter bis zur Gegenwart vom 14. bis 17. April in Bamberg, Vierteljahresschrift für Sozial- und Wirtschaftsgeschichte, Beiheft 114, Stuttgart 1994, S. $215 \mathrm{f}$. 
(VEB), Steuern der Bevölkerung und privater Betriebe spielten im Vergleich dazu keine Rolle. ${ }^{984}$

In der DDR besaßen rechtsstaatliche Steuerprinzipien keine Geltung. Eine Besteuerung der Steuersubjekte nach den Grundsätzen der Allgemeinheit, der Gleichmäßigkeit und der Beachtung der Leistungsfähigkeit wurde ausdrücklich abgelehnt. ${ }^{985}$ Aus dem Staatsverständnis ergab sich, dass eine sozialistische Regierung ihre Steuerpolitik nicht nach den überlebten Grundsätzen einer bürgerlich-kapitalistischen Klassengesellschaft ausrichten könne; denn dies behindere die Überwindung der privatwirtschaftlichen Wirtschaftsordnung und sabotiere den angestrebten Aufbau eines sozialistischen Wirtschaftssystems. Die der Privatwirtschaft auferlegten Steuern waren ihrer Art nach aus der Steuergesetzgebung des Deutschen Reiches aus dem Nationalsozialismus übernommen worden. Diese Steuern wurden jedoch vom Gesetzgeber der DDR sozialistisch umgeformt, um sie als Klassenkampfinstrument zur Fesselung, Verdrängung und Liquidation der Privatwirtschaft nutzen zu können. ${ }^{986}$

Entsprechend betrug der Höchstsatz der Einkommensteuer auf Einkünfte aus Unternehmertätigkeit und Kapitalnutzung 90 Prozent und der Körperschaftsteuersatz für die noch übriggebliebenen Kapitalgesellschaften 95 Prozent. Mit diesen hohen Steuersätzen sollte der Privatwirtschaft Kapital entzogen und dieses durch Umverteilung über den Staatshaushalt zum Aufbau sozialistischer Staatsbetriebe verwendet werden. ${ }^{987}$

\subsection{Die verfassungsrechtlichen Grundlagen in der Bundesrepublik Deutschland}

Im Gegensatz zur Weimarer Reichsverfassung enthält das Grundgesetz vom 23. Mai 1949 (GG) keine spezielle finanz- und steuerrechtliche Grundrechtsnorm mehr, in

984 Hacke, Constanze: Blick nach Europa. In: Bundeszentrale für politische Bildung. Informationen zur politischen Bildung vom 24.10.2012, auf: http://www.bpb.de/izpb/147109/ blick-nach-europa?p=all (letzter Zugriff 17.02.2017), S. $20 \mathrm{f}$.

985 Buck, Hannsjörg: „Steuern“ in: Eppelmann, Rainer u.a. (Hrsg.): Lexikon des DDR-Sozialismus. Das Staats- und Gesellschaftssystem der Deutschen Demokratischen Republik, Paderborn 1996, S.616-619, S.616. Da in der DDR rechtsstaatliche Steuerprinzipien keine Geltung besaßen, entfällt jeder Bezug zur Thematik, weshalb hier nicht weiter auf die Verhältnisse in der DDR eingegangen wird.

986 Ebd., S. 617.

987 Ebd., S. 617. 
der die Prinzipien der Allgemeinheit ${ }^{988}$, der Gleichmäßigkeit und der Leistungsfähigkeit explizit festgeschrieben worden sind. Eine inhaltlich dem Art. 134 der Weimarer Verfassung entsprechende Vorschrift in das Grundgesetz aufzunehmen, war von den Schöpfern dieser Verfassung offensichtlich nicht beabsichtigt. ${ }^{989}$ Die während der nationalsozialistischen Diktatur regelmäßig vorkommende Missachtung der Rechte Einzelner war Grund genug, den Grundrechten des Grundgesetzes gegenüber denjenigen der Weimarer Reichsverfassung eine stärkere Geltungskraft zu verleihen, um damit der Allmacht des Staates wirksame Schranken zu setzen. Die Absicht der Verfassungsgeber war insbesondere, die Stellung des Einzelnen allein durch seine Rechte zu definieren. Damit wollte man dem Missbrauch des Pflichtgedankens der nationalsozialistischen Diktatur vorbeugen, unter der die subjektiven Rechte des Einzelnen nur in geringem Maße Anerkennung gefunden hatten. Die Verfasser des Grundgesetzes wichen deshalb ausdrücklich von dem Weimarer Vorbild ab und verzichteten auf die Aufnahme von verallgemeinernden Grundpflichten sozialer und gesellschaftspolitischer Natur. Durch diese Verfahrensweise entfiel ein Verfassungsartikel, der den Grundsatz der Gleichmäßigkeit und der Leistungsfähigkeit der Besteuerung ausdrücklich festgeschrieben hätte. ${ }^{990}$

Aus dem in Art. 3 Abs. 1 GG kodifizierten Gleichheitssatz - „Alle Menschen sind vor dem Gesetz gleich “ - wird das Gebot der Steuergerechtigkeit hergeleitet. ${ }^{991}$ Entsprechend liegt das Schwergewicht der Überprüfung steuerrechtlicher Normen durch das Bundesverfassungsgericht beim allgemeinen Gleichheitssatz. ${ }^{992}$ Dabei hat das Bundesverfassungsgericht ständig entschieden, dass der Gesetzgeber , an den Grundsatz der Steuergerechtigkeit gebunden ist, der sich aus dem Gleichheitssatz ergibt. ${ }^{{ }^{1993}}$ Der Gesetzgeber darf nach seinem Ermessen entscheiden, welche

988 Der Grundsatz der Allgemeinheit der Besteuerung wird heute nicht als selbstständiger Grundsatz erfasst, da er Ausfluss des Grundsatzes der Gleichmäßigkeit ist; vgl. z. B. Schneider, Dieter: Steuerbilanzen, S. 16; Hey, Johanna: $\$ 3$ Steuersystem und Steuerverfassungsrecht, S. $66 \mathrm{ff}$.

989 Davidsohn, Lars: Verfassungsrechtliche Würdigung, S. 44; Oechsle, Klaus: Die steuerlichen Grundrechte, S. 186.

990 Davidsohn, Lars: Verfassungsrechtliche Würdigung, S.45-47; Oechsle, Klaus: Die steuerlichen Grundrechte, S. 189; Tipke, Klaus: Die Steuerrechtsordnung, S. 491.

991 Hey, Johanna: $\$ 3$ Steuersystem und Steuerverfassungsrecht, S.93; Tipke, Klaus: Die Steuerrechtsordnung, S. 290.

992 Hey, Johanna: Rechtsprechung des Bundesverfassungsgerichts zum Steuerrecht im Wandel?, in: Piltz, Detlev J./Günkel, Manfred/Niemann, Ursula (Hrsg.): Steuerberater-Jahrbuch 2007/2008, S. 19-58, S. 33.

993 Tipke, Klaus: Steuerrecht als Wissenschaft, in: Tipke, Klaus u.a. (Hrsg.): Gestaltung der Steuerrechtsordnung. Festschrift für Joachim Lang zum 70. Geburtstag, Köln 2010, 
„Elemente der zu ordnenden Lebensverhältnisse er als maßgebend ansieht, sie im Recht gleich oder verschieden zu behandeln. Der Gesetzgeber darf selbst diejenigen Sachverhalte auswählen, die er als gleich ansehen will. " ${ }^{\text {"994 }}$ Vom Bundesverfassungsgericht ist nur die Einhaltung der äußersten Grenzen der gesetzgeberischen Freiheit („Willkürverbot“) zu prüfen, nicht jedoch, „ob der Gesetzgeber im Einzelfall die jeweils zweckmäßigste, vernünftigste und gerechteste Lösung gefunden hat.“995

Die verhaltene Rechtsprechung, die sich das Bundesverfassungsgericht in den 1960er und 1970er Jahren auferlegt hatte, veränderte sich seit Beginn der 1980er Jahre mit wesentlichen Beiträgen zur Durchsetzung der systemtragenden Prinzipien eines verfassungskonformen Steuerrechts. Dabei ließ das Bundesverfassungsgericht keine Zweifel, „dass der gesetzgeberische Gestaltungsspielraum verfassungsrechtlich durch die Prinzipien der finanziellen Leistungsfähigkeit und der Folgerichtigkeit begrenzt ist. ${ }^{\text {"9966 }}$ Das Bundesverfassungsgericht gewährleistete nachdrücklich die Steuerfreiheit des Existenzminimums, den Schutz von Ehe und Familie, erteilte reinen Gegenfinanzierungsmaßnahmen eine deutliche Absage und verstärkte die Anforderungen an den rechtsstaatlichen Vertrauensschutz. ${ }^{997}$

Mit dem Vermögensteuerbeschluss vom 22. Juni $1995^{998}$ ging der Zweite Senat von einem Eingriff in die Eigentümerfreiheit aus und erklärte damit eine Belastungsobergrenze. ${ }^{999}$ Zehn Jahre später distanzierte sich der Zweite Senat des Bundesverfassungsgerichts im Beschluss vom 18. Januar 2006 ${ }^{1000}$ von der Idee einer Belastungsobergrenze mit der Begründung, die Aussagen des Vermögensteu-

S. 21-56, S. 49, mit Hinweis auf Urteilsnachweise.

994 Tipke weist mit diesem Passus auf BVerfGE 13, 202, 21, 27; vgl. Tipke, Klaus: Steuerrecht als Wissenschaft, S. 50.

995 Ebd., S. 50; Tipke weist darauf hin, dass diese Rechtsprechung zurückgeht auf BVerfGE $1,52,4,18,26,310$.

996 Hey, Johanna: $\$ 3$ Steuersystem und Steuerverfassungsrecht, S. 91.

997 Ebd., S.91.

998 BVerfGE 93, 121.

999 Sogenannter Halbteilungsgrundsatz: „Die Vermögensteuer darf zu den übrigen Steuern auf den Ertrag nur hinzutreten, soweit die steuerliche Gesamtbelastung des Soll-Ertrages bei typisierender Betrachtung von Einnahmen, abziehbaren Aufwendungen und sonstigen Entlastungen in der Nähe einer hälftigen Teilung zwischen privater und öffentlicher Hand verbleibt." Vgl. BVerfGE 93, 121, LS 3. Nach Klaus Vogel hatte der „Halbteilungsgrundsatz" den Zweck, den Machtzuwachs und das Selbstbedienungsverständnis der Politiker zu beschneiden. Jedoch entspricht dieses Politikverständnis nicht dem Demokratieverständnis des Grundgesetzes; vgl. Vogel, Klaus: Verfassungsrechtsprechung zum Steuerrecht. Vortrag gehalten vor der Juristischen Gesellschaft zu Berlin am 16. September 1998, Berlin/New York 1999, S. 1-23, S. 23.

1000 BVerfGE 115, 97. 
erbeschlusses seien den Besonderheiten der zur Einkommensteuer hinzutretenden Vermögensteuer geschuldet. Damit hat sich das Gericht „um einen Ausgleich zwischen demokratisch legitimiertem Steuerstaat und individuellem Schutz durch das freiheitliche Übermaßverbot bemüht. ${ }^{\text {“1001 }}$

Der neue Staatszweck in der Bundesrepublik Deutschland ab 1949, dem das Steuerrecht angepasst wurde, war Lenkung und Steuerung, weniger die Umverteilung. Die Funktion der Steuer hatte sich verändert: Die Frage war nicht mehr - oder allenfalls nachrangig -, ob die Steuern "gerecht" waren, sondern, ob diese wirtschaftspolitisch „richtig“ oder "nicht richtiger“, „hilfreicher“ oder „nicht hilfreicher" waren. ${ }^{1002}$ Damit verlor die Einkommensteuer als Instrument der sozialen Umverteilung an Gewicht, verglichen mit der Aufgabe, die ihr Finanzminister Matthias Erzberger 1920 zugewiesen hatte. ${ }^{1003}$ Die Fiskalpolitik

ermöglichte es, die steuerlichen Gestaltungswirkungen für die wirtschaftspolitischen Aufgaben und Ziele des Staates fruchtbar zu machen [...], indem diese, gestaltende Wirkungsweise wirtschafts-, währungs-, konjunktur- und sozialpolitischen Zwecken dienstbar gemacht wurde. ${ }^{1004}$

Die Verfassung verbietet es grundsächlich nicht, mithilfe der Steuerpolitik Lenkungszwecke zu verfolgen. In der Legaldefinition des Steuerbegriffs gemäß $₫ 3$ Abs. 1 Satz 1 Halbsatz 2 Abgabenordnung (AO) heißt es: „[D]ie Erzielung von Einnahmen kann Nebenzweck sein." Damit hat der Gesetzgeber beim Erlass steuerlicher Vorschriften die Möglichkeit, außerfiskalische Zwecke zu verfolgen. Der Steuergesetzgeber ist nach ständiger Rechtsprechung des Bundesverfassungsgerichts nicht gehindert, außerfiskalische Förderungs- und Lenkungsziele aus Gründen des Gemeinwohls zu verfolgen, und hat dabei eine große Gestaltungsfreiheit. ${ }^{1005}$ Weder der fiskalische Zweck noch die Verwendung der Steuern geben der Steuerbelastung Anknüpfungspunkte oder ziehen ihr Grenzen. ${ }^{1006}$

1001 Hey, Johanna: $\$ 3$ Steuersystem und Steuerverfassungsrecht, S. 115-118, S. 118.

1002 Schremmer, Eckart: Über „gerechte Steuern“, S. 27.

1003 Ebd., S. 38.

1004 Birk, Dieter: Das Leistungsfähigkeitsprinzip, S. $72 \mathrm{f}$.

1005 Ständige Rechtsprechung vgl. BVerfG v. 22.06.1995-2 Bvl. 37/91; BVerfGE 93, 121 (136); BVerfG v. 30.09.1998-2 BvR 1818/91; BVerfGE 99, 88 (95); BVerfG. v. 04.12.2002-2 BvR 400/98; BVerfGE 107, 27 (47), BFH v. 27.09.2007, DStR 2007, 2256 (2259); BVerfG v. 07.11.2006-1BvL 10/02, FR 2007, 338 (340); zuletzt BVerfGE 117, 1 (31) betr. Erbschaftsteuer und BVerfGE 122, 210 (231) betr. Entfernungspauschale.

1006 Drüen, Klaus-Dieter: Die Bruttobesteuerung von Einkommen als verfassungsrechtliches Vabanquespiel, in: Steuer und Wirtschaft 85 (2008), S. 3-14, S. 8 mit der dort 
Abweichungen vom Leistungsfähigkeitsprinzip bedürfen der Rechtfertigung durch „Gründe des Gemeinwohls“. Steuervergünstigungen müssen sich „gemeinwohlbezogen "1007 rechtfertigen lassen, Sonderbelastungen durch Sozialzwecksteuern, Abzugsverbote und andere Steuerverschärfungen müssen durch das Gemeinwohl gerechtfertigt sein. Das Leistungsfähigkeitsprinzip darf dabei eingeschränkt, jedoch nicht ausgeschaltet sein. ${ }^{1008}$ Der verfolgte Lenkungszweck muss gleichheitsgerecht ausgestaltet $\operatorname{sein}^{1009}$ und gebietet auch die Beachtung des Verhältnismäßigkeitsprinzips ,unter den Gesichtspunkten der Geeignetheit der Regelung, deren Notwendigkeit und Angemessenheit im engeren Sinn. ${ }^{\text {"1010 }}$

\subsection{Zum Grundsatz der Gleichmäßigkeit der Besteuerung}

\subsubsection{Bedeutung und Inhalt des allgemeinen Gleichheitssatzes und die Rechtfertigung von Sozialzwecknormen}

Der Gleichheitsgrundsatz (Art. 3 Abs. 1 GG) als „Schlüsselbegriff der Gerechtigkeit" gilt als Maßstab für die Gleichmäßigkeit der Besteuerung, ${ }^{1011}$ die nach der Rechtsprechung des Bundesverfassungsgerichts verletzt ist, „wenn sich ein aus der Natur der Sache ergebender oder sonstwie sachlich einleuchtender Grund für die gesetzliche Differenzierung oder Gleichbehandlung nicht finden lasse, kurzum, wenn die Bestimmung willkürlich sei. ${ }^{\text {"1012 }}$

Der Gesetzgeber ist gemäß Art. 3 Abs. 1 GG nach ständiger Formulierung des Bundesverfassungsgesetzes verpflichtet - bei steter Orientierung an dem Gerechtigkeitsgedanken -, wesentlich Gleiches gleich, wesentlich Ungleiches verschieden zu behandeln. Das Gebot dieser genannten Rechtsetzungsgleichheit stellt an den Gesetzgeber einen am Wertesystem des Grundgesetzes orientierten Gerechtig-

angegebenen Rechtsprechung.

1007 BVerfGE 93, 121 (148).

1008 Hey, Johanna: $\$ 3$ Steuersystem und Steuerverfassungsrecht, S. 101.

1009 BVerfG v. 20.04.2004; BVerfGE 110, 274 (293).

1010 Weber-Grellet, Heinrich: Steuern, S. 174; vgl. auch Glaser, Andreas: Verfassungs- und unionsrechtliche Grenzen steuerlicher Lenkung, in: Steuer und Wirtschaft 89 (2012),

S. 168-181, S. 173.

1011 Weber-Grellet, Heinrich: Steuern, S. 33.

1012 Tipke, Klaus: Die Steuerrechtsordnung, S. 295 mit der dort zitierten Rechtsprechung. 
keitsauftrag dar; maßgeblich hierfür sind der tatsächliche Belastungserfolg sowie die Einhaltung des Folgerichtigkeitsgebotes. Zwar habe der Gesetzgeber bei der Auswahl des Steuergegenstandes und der Bestimmung des Steuersatzes einen weitreichenden Gestaltungspielraum, er müsse jedoch die einmal getroffene Belastungsentscheidung folgerichtig umsetzen. Dabei wird sein Belastungsspielraum „Vor allem durch zwei eng miteinander verbundene Leitlinien begrenzt: durch die Ausrichtung der Steuerlast an den Prinzipien der finanziellen Leistungsfähigkeit und der Folgerichtigkeit.“1013

Der Gleichheitssatz verlangt zudem den gleichmäßigen Vollzug (Rechtsanwendungsgleichheit) der Steuergesetze als wesentliches Moment der Steuergerechtigkeit. Die Belastung ist für alle Steuerpflichtigen nur erträglich, wenn zu erkennen ist, dass alle in derselben Situation denselben Eingriff zu dulden haben. ${ }^{1014}$ Das Bundesverfassungsgericht hat im Zinsurteil vom 27. Juni 1991 diese Bedeutung deutlich herausgestellt:

Der Gleichheitssatz verlangt für das Steuerrecht, dass die Steuerpflichtigen durch ein Steuergesetz rechtlich und tatsächlich gleich belastet werden. Die Besteuerungsgleichheit hat mithin als ihre Komponenten die Gleichheit der normativen Steuerpflicht ebenso wie die Gleichheit bei deren Durchsetzung in der Steuererhebung. ${ }^{1015}$

Die steuerliche Lastengleichheit fordere, „dass das materielle Steuergesetz die Gewähr seiner regelmäßigen Durchsetzbarkeit so weit wie möglich in sich selbst trägt."1016 Der Gleichheitssatz sei verletzt, wenn das „Ziel der Gleichheit im Belastungserfolg prinzipiell nicht zu erreichen" sei. ${ }^{1017}$

Der Gleichheitssatz verlangt also, dass die Steuerpflichtigen durch ein Steuergesetz rechtlich und tatsächlich gleich belastet werden. Der Verfassung wird entsprechend mit der Rechtsetzungsgleichheit nicht Genüge getan, „vielmehr fordert sie die Rechtsanwendungsgleichheit in ihrer Realisierung durch die gleichmäßige Besteuerung für alle, die den Tatbestand des Gesetzes erfüllen. "1018

Schon die Kameralisten vertraten den Leitgedanken, dass eine gleichmäßige und gerechte Verteilung der Steuerlasten nur dann zu erreichen ist, wenn die einzelnen

1013 Hey, Johanna: $\$ 3$ Steuersystem und Steuerverfassungsrecht, S. 96.

1014 Weber-Grellet, Heinrich: Steuern, S. 36.

1015 BVerfGE 84, S. 239.

1016 Ebd., S. 271.

1017 Ebd., S. 272.

1018 Isensee, Josef: Vom Beruf unserer Zeit, S. 8. 
Steuerarten aufeinander abgestimmt werden. ${ }^{1019} \mathrm{Zu}$ bedenken ist, dass der Umfang des individuellen Verbrauchs insbesondere von der Größe der Familie beeinflusst wird und relativ unabhängig von der Einkommenshöhe eines Steuerpflichtigen sein dürfte. Infolgedessen wirken sich die auf den notwendigen Lebensbedarf lastenden Steuern meistens zu Ungunsten der weniger wohlhabenden Bevölkerung aus. ${ }^{1020}$ Da bei den direkten Steuern - insbesondere der Einkommensteuer - in besonderem Maße steuerliche Leistungsmerkmale berücksichtigt werden können, können damit weniger erwünschte Belastungswirkungen anderer Steuerarten weitgehend kompensiert werden. ${ }^{1021}$

Bei der verfassungsrechtlichen Beurteilung des Grundsatzes der GleichmäBigkeit der Besteuerung ist auch zu beachten, dass der steuerpflichtige Bürger Anspruch auf eine dem Leistungsfähigkeitsprinzip und dem Sozialstaatsprinzip entsprechende Verteilung der Steuerlasten hat. Der insbesondere in Art. 20 Abs. 1 GG ausformulierte Sozialstaatsgedanke „ist nach heute einhelliger Meinung Verfassungsnorm mit verpflichtender Wirkung für alle Staatsgewalten. ${ }^{\text {"1022 Eine }}$ ungleichmäßige Besteuerung widerspricht nicht nur dem aus Art. 3 Abs. 1 GG hergeleiteten Grundsatz der Steuergerechtigkeit, sondern gleichzeitig dem im GG verankerten Sozialstaatsprinzip; denn eine gerechte Steuerlastverteilung kann ebenso durch den Sozialstaatsgedanken begründet werden. ${ }^{1023}$

Im Grunde wurde erst durch die Sozialgesetzgebung der Bundesrepublik Deutschland

das Postulat Wagners erfüllt, soziale Finanz- und Steuerpolitik nicht nur im Besteuerungssystem, sondern in einer Kombination von Steuern und Transferleistungen zu verwirklichen. Heute ist anerkannt, daß soziales Nehmen (Steuern) und soziales Geben (Sozialleistungen) einander bedingen. ${ }^{1024}$

1019 Mann, Fritz Karl: Grundsätze der Besteuerung, in: Spiethoff, Arthur (Hrsg.): Schmollers Jahrbuch für Gesetzgebung, Verwaltung und Volkswirtschaft im Deutschen Reiche, 50. Jg., 1. Halbband, München/Leipzig 1926, S. 55-82, S. 82.

1020 Davidsohn, Lars: Verfassungsrechtliche Würdigung, S. 183.

1021 Ebd., S. 183-187; Wagner, Adolph: Finanzwissenschaft 2. Teil, S. 370.

1022 Lehner, Moris: Einkommensteuerrecht und Sozialhilferecht. Bausteine zu einem Verfassungsrecht des sozialen Steuerstaates (Beiträge zum öffentlichen Recht 5), Tübingen 1993, S. 341.

1023 Kirchhof, Paul: Steuergleichheit, in: Steuer und Wirtschaft 61 (1984), S. 297-314, S. 306.

1024 Birk führt hierzu aus: „Der Gedanke der sozialen Besteuerung ist [...] in ganz erheblichem Maße ineffektiv, wenn an einem Transferstrom, dem steuerlichen Wertetransfer, festgehalten wird. Denn soziale Entlastungen sind nur insoweit möglich, soweit die wirtschaftliche Lage des einzelnen Steuerzahlungen überhaupt ermöglicht. Sie versagen also in den Fällen, in denen soziale Hilfe am dringendsten benötigt wird. Den Anspruch 
Staatliches Nehmen muss die Freiheit des Betroffenen wahren, staatliches Geben seine soziale Existenz sichern. Gleichheit der Bürger führt somit bei der Auferlegung öffentlicher Lasten zu einer die Einnahmen- und Ausgabenseite umgreifenden Gleichheit. ${ }^{1025}$

$\mathrm{Zu}$ den Aufgaben des Sozialstaats gehört insbesondere die Sicherung eines menschenwürdigen Existenzminimums, das im Steuerrecht vor Steuerbelastung geschützt werden muss. ${ }^{1026}$ Die erste wirtschaftstheoretische Begründung der Steuerfreiheit des Existenzminimums lieferte die physiokratische Lehre vom „produit net“ (Reinertrag). Die Naturrechtslehre bildete das ethische Fundament der Steuerfreiheit des Existenzminimums, auf das sich heute noch deren Rechtfertigung aus den Menschenrechten gründet. ${ }^{1027}$ Dass der Staat jedem Bürger das Existenzminimum gewährleistet, verlangen nach der Verfassung nicht nur das Sozialstaatsprinzip, sondern auch die Menschenwürde (Art. 1 Abs. 1 GG) und das Freiheitsprinzip (Art. 2 Abs. 1 GG). ${ }^{1028}$

\subsubsection{Zum Grundsatz der Gleichmäßigkeit der Besteuerung am Beispiel der Unternehmensbesteuerung}

Der Gleichheitssatz in Art. 3 Abs. 1 GG wird durch das BVerfG im Hinblick auf die Gleichmäßigkeit der Besteuerung durch das Gebot konkretisiert, „wesentlich Gleiches gleich und wesentlich Ungleiches ungleich zu behandeln. " ${ }^{1029}$ Das Kernproblem liegt entsprechend in der Bewertung von Sachverhalten als gleich oder ungleich. ${ }^{1030}$ Am Beispiel der Unternehmensbesteuerung soll aufgezeigt werden, wie schwierig es sich darstellt, das Gebot dieser Rechtsetzungsgleichheit zu erreichen.

an ein soziales Transferverhältnis zwischen Bürger und Staat konnte die Steuer von vornherein nicht vollständig erfüllen. Es mußte ein ergänzender Geldleistungsstrom geschaffen werden, der die sozialen Defizite auffängt, die im Besteuerungssystem keine Berücksichtigung finden können." Birk, Dieter: Das Leistungsfähigkeitsprinzip, S. 115, Anm. 68.

1025 Kirchhof, Paul: Die Leistungsfähigkeit des Steuerrechts - Steuerrecht und Verfassungsrecht, in: Steuer und Wirtschaft 88 (2011), S. 365-371, S. 366.

1026 Tipke, Klaus: Die Steuerrechtsordnung, S. 97.

1027 Treisch, Corinna: Existenzminimum und Einkommensbesteuerung. Dogmengeschichte, Analyse der Besteuerungsprinzipien und Regelungsvorschlag, Aachen 1999, S.7.

1028 Tipke, Klaus: Die Steuerrechtsordnung, S. 420.

1029 BVerfG 2009a, 17; BVerfG 2009b, 265; BVerfG 2010a, 277; BVerfG 2010b, 416; BVerfG 2012, 2720.

1030 Birk, Dieter: Das Leistungsfähigkeitsprinzip, S. 157. 


\subsubsection{Die Entwicklung des Körperschaftsteuersystems in der Bundesrepublik Deutschland}

Die Nachkriegszeit bis zur Einführung des Anrechnungsverfahrens 1977

Parallel zu den Bestrebungen, das klassische Körperschaftsteuersystem neutral zu gestalten, wurde das dualistische System nach der Währungsreform im Jahr 1948 grundsätzlich infrage gestellt. Der von der Finanzverwaltung einberufene Ausschuss legte im Jahr 1949 drei Entwürfe einer Betriebsteuer vor. ${ }^{1031}$ Alle drei Betriebsteuerentwürfe umfassten als Kreis der Steuerpflichtigen alle gewerblichen Betriebe ohne Rücksicht auf ihre Rechtsform. Nach den Trennungsbestrebungen von Einkommensteuer und Körperschaftsteuer ab dem Jahr 1891 stand die Zusammenfassung beider Steuerarten wieder zur Diskussion; Steuertarif und Besteuerungsgrundlage sollten sich nicht mehr unterscheiden. Alle drei Entwürfe einer Betriebsteuer wurden jedoch nie Gesetz. ${ }^{1032}$

Durch die sogenannte Kleine Steuerreform im Jahr $1953^{1033}$ wurde erstmals ein gespaltener Körperschaftsteuertarif kodifiziert, der die Doppelbelastung ausgeschütteter Gewinne minderte. Damit wurde es lange Zeit ruhig um die grundsätzliche Rechtfertigung des dualistischen Unternehmensteuersystems, bis Ende der 1960er-Jahre die Reformdiskussion wieder neu angestoßen wurde. ${ }^{1034}$ Die Debatte ging weit über die Beseitigung der Doppelbelastung hinaus, denn das bestehende Körperschaftsteuersystem wurde in seiner Grundstruktur infrage gestellt. Die Umgehung der Doppelbelastung war neben der Ausschöpfung steuerlicher Optimierungsmöglichkeiten das primäre Ziel der Steuerpflichtigen. Die Vereinbarung von Leistungsvergütungen zwischen Gesellschaft und Gesellschafter durch Geschäftsführergehälter, Darlehens- oder Miet- und Pachtzinsen stellten bei den Gesellschaften Betriebsausgaben dar, was zu einer Verminderung des steuerpflichtigen Einkommens führte. Unangemessene Leistungsvergütungen bewirkten wiederum, dass der Gesetzgeber die Umgehung der Doppelbelastung durch das Institut der „verdeckten Gewinnausschüttung“ zu verhindern suchte.

Unabhängig von der Wahl einer Rechtsform wurde die Entwicklung von Mischformen zur Vermeidung der Doppelbelastung intensiviert. Als typische Gestaltungsvarianten sollen hier die $\mathrm{GmbH} \& \mathrm{Co}$. KG, die Betriebsaufspaltung und

1031 o. V.: Betriebsteuerausschuss der Verwaltung der Finanzen: Bericht und Gesetzentwürfe zur Betriebsteuer, in: Steuer und Wirtschaft 1949, Sp. 929-1068, Sp. 929 ff., insb. Sp. $1021 \mathrm{ff}$.

1032 Potthast, Thilo: Die Entwicklung der Körperschaftsteuer, S. 74-79.

1033 Gesetz zur Änderung steuerlicher Vorschriften und zur Sicherung der Haushaltsführung vom 24.06.1953, BGBl. 1953 I, S. 413.

1034 Potthast, Thilo: Die Entwicklung der Körperschaftsteuer, S. 79. 
die Stille Gesellschaft erwähnt werden. Um eine Doppelbelastung zwischen einer Kapitalgesellschaft und einer anderen Kapitalgesellschaft zu verhindern, kamen das Schachtelprivileg ${ }^{1035}$ und die körperschaftsteuerliche Organschaft zur Anwendung, um die Mehrfachbelastungen im Konzernbereich zu vermeiden. ${ }^{1036}$

In der Reformdiskussion wurde eine Reihe von Alternativen ${ }^{1037}$ zum klassischen Körperschaftsteuersystem herausgearbeitet, die Ausgangspunkt für die Ablösung des bisherigen Steuersystems durch das Anrechnungsverfahren im Jahr 1977 waren, das eine Doppelbelastung ausgeschütteter Gewinne verhindern sollte.

\section{Das körperschaftsteuerliche Anrechnungsverfahren von 1977 bis 2000}

Der Einführung des Anrechnungsverfahrens ging eine zehnjährige, intensiv geführte wissenschaftliche Diskussion voraus. Die Bundesregierung schloss sich im Wesentlichen den Vorstellungen der Steuerreformkommission von 1971 an, die zu dem Ergebnis gekommen war, dass die ertragsteuerliche Doppelbelastung bei der Körperschaftbesteuerung zu erheblichen Nachteilen geführt hatte. ${ }^{1038}$ Der Gesetzgeber entschied sich für die Vollanrechnung der Körperschaftsteuer auf den ausgeschütteten Gewinn, kombiniert mit einem ermäßigten Ausschüttungsteuersatz auf Körperschaftsebene. Die körperschaftsteuerliche Vorbelastung wurde bei einer Ausschüttung auf eine einheitliche Ausschüttungsbelastung (zunächst 36 Prozent, zuletzt 30 Prozent) herab- bzw. heraufgesetzt und dann durch Anrechnung auf die Einkommen- bzw. Körperschaftsteuer und gegebenenfalls durch Vergütung vollständig neutralisiert. ${ }^{1039}$ Damit stellte die Körperschaftsteuer eine Art Vorauszahlung auf die Einkommensteuer dar, die der Anteilseigner auf seine Steuerschuld anrechnen konnte. Dies galt jedoch nicht für ausländische Anteilseigner. Diese waren nicht anrechnungsberechtigt, sodass die deutsche Körperschaftsteuer für sie in Deutschland eine endgültige Belastung darstellte. ${ }^{1040}$

Rechtsformneutralität konnte das Anrechnungsverfahren nur hinsichtlich ausgeschütteter Gewinne für anrechnungsberechtigte Steuerpflichtige gewährleisten.

1035 Das Schachtelprivileg ist ein Instrument zur Vermeidung von Mehrfach- oder Doppelbelastungen, die sich bei der Verschachtelung von Kapitalgesellschaften ergeben.

1036 Potthast, Thilo: Die Entwicklung der Körperschaftsteuer, S. 127.

1037 Ebd., S. 114-120.

1038 Gutachten der Steuerreformkommission 1971 (Schriftenreihe des Bundesministeriums der Finanzen 17), Bonn 1971, S. 303-314, Rz. 40-93.

1039 Körperschaftsteuerreformgesetz vom 31.08.1976, BStBl 1976 I, S. 2597; Hey, Johanna: § 11 Körperschaftsteuer, in: Tipke, Klaus/Lang, Joachim (Hrsg.): Steuerrecht, fortgeführt von Roman Seer u. a., 22. Aufl., Köln 2015, S.669-716, S. 672.

$1040 \$ 50$ Abs. 5 EStG 1977; $\$ 50$ Abs. 1 Nr. 2 und $\$ 51$ KStG 1977. 
Schüttete eine Kapitalgesellschaft ihre Gewinne vollständig aus, ergaben sich bei sonst gleicher Gewinnsituation keine Unterschiede hinsichtlich der Steuerbelastung von Anteilseignern einer Kapitalgesellschaft und den Gesellschaftern einer Personengesellschaft bzw. einem Einzelunternehmer. ${ }^{1041}$ Damit wurde das Ziel, Rechtsformunterschiede zu beseitigen, zumindest im Ertragsteuerbereich nahezu erreicht. Hinsichtlich einbehaltener Gewinne kam es nur zu einer Annäherung der Steuerbelastung, was dazu führte, dass Steuerpflichtige verschiedene Möglichkeiten der Steueroptimierung im Anrechnungsverfahren ausnutzten. ${ }^{1042}$ Lag z. B. die persönliche Einkommensteuer eines Gesellschafters unter dem Spitzensteuersatz, so verleitete das Anrechnungsverfahren selbst dann die Ausschüttung der Gewinne, wenn die Mittel in der Kapitalgesellschaft benötigt wurden. Die Vorteile des „Schüttaus-hol-zurück-Verfahrens“ sowie der „negative“ Ausländereffekt führten zu einer zunehmenden Beliebtheit der Gesellschafter-Fremdfinanzierung. Die Attraktivität der Fremdfinanzierung bewirkte ein krasses Missverhältnis zwischen Eigen- und Fremdkapitalausstattung und führte zu Steuerausfällen, was den Gesetzgeber wiederum zwang zu reagieren.

In den 1990er-Jahren war eine deutliche Zunahme der Änderungen des Körperschaftsteuergesetzes zu verzeichnen, die durch gegenläufige Entwicklungen geprägt waren. Zum einen erfolgte eine Abkehr von der Substanzbesteuerung durch die Aussetzung der Vermögensteuer und die Abschaffung der Gewerbesteuer vom Kapital. Zum anderen sah sich die Bundesregierung durch den internationalen Steuersenkungswettbewerb dazu genötigt, die Kopplung des Körperschaftsteuersatzes für thesaurierte Gewinne und des Einkommensteuerspitzensatzes aufzugeben. ${ }^{1043}$

Da wegen des internationalen Steuersenkungswettbewerbs das Problem der Tarifspreizung nicht gelöst werden konnte, war eine Unternehmensteuerreform zwingend erforderlich. Dazu kam, dass die fortschreitende Globalisierung insbesondere die Schwächen des Anrechnungsverfahrens im Umgang mit Auslandssachverhalten zum Problem werden ließ. Das Anrechnungsverfahren war auf die Anrechnung inländischer Körperschaftsteuer beschränkt, ${ }^{1044}$ worin ein Verstoß

1041 Scheffler, Wolfram: Besteuerung von Unternehmen, Bd. I: Ertrag-, Substanz- und Verkehrsteuern, 13. Aufl., Heidelberg 2016, S. 244; Hey, Johanna: § 7 Einführung in das besondere Steuerschuldrecht, in: Tipke, Klaus/Lang, Joachim (Hrsg.): Steuerrecht, fortgeführt von Roman Seer u.a., 22. Aufl., Köln 2015, S. 247-273, S. 257.

1042 Potthast, Thilo: Die Entwicklung der Körperschaftsteuer, S. 148-160.

1043 Ebd., S. 179.

1044 Ausschüttungen an ausländische Anteilseigner wurden benachteiligt, da diesen die Anrechnung der in Deutschland gezahlten Körperschaftsteuer nicht möglich war. 
gegen die Kapitalverkehrsfreiheit gesehen wurde. ${ }^{1045}$ Dies war ein maßgebliches Motiv, weshalb das körperschaftsteuerliche Anrechnungsverfahren im Jahr 2000 in Deutschland aufgegeben wurde. ${ }^{1046}$

\section{Das klassische System mit pauschaler Entlastung auf Anteilseignerebene seit 2001}

Im Jahr 1998 wurde von der Bundesregierung eine unabhängige Kommission eingesetzt, um eine grundlegende Reform der Unternehmensbesteuerung zu erarbeiten. In den Brühler Empfehlungen hieß es:

Ziel ist eine rechtsformneutrale Unternehmensbesteuerung, bei der alle Unternehmenseinkünfte mit höchstens $35 \%$ besteuert werden. [...] Investitionen in Deutschland sollen sich lohnen. [...] Aus diesem Grund ist eine deutliche Senkung der Steuersätze für Unternehmen erforderlich. ${ }^{1047}$

Auf der Grundlage dieser Empfehlungen wurde durch das Steuersenkungsgesetz ${ }^{1048}$ das seit 1977 bestehende körperschaftsteuerliche Anrechnungsverfahren durch ein klassisch-dualistisches Körperschaftsteuersystem mit Teilentlastungsmechanismus, das allgemein als „Halbeinkünfteverfahren“ bezeichnet wird, ersetzt. ${ }^{1049}$

Die Gewinne einer Körperschaft werden nun - wie schon in den Jahren vor 1977 - sowohl auf der Ebene der Gesellschaft als auch auf der Ebene des Gesellschafters doppelt besteuert. Dabei lassen Gewinnausschüttungen einer Körperschaft die Körperschaftsteuer unberührt. ${ }^{1050}$ Die Vorbelastung auf der Ebene der ausschüttenden Körperschaft wird aber beim Empfänger berücksichtigt. Der Anteilseigner (Ausschüttungsempfänger) hatte seinen Gewinnanteil aus der Beteiligung (Dividende)

1045 Meilicke, Wienand: Nachlese zum EuGH-Urteil vom 06.03.2007 - Rs. C-292/04, in: Der Betrieb 2007, S. 650 f., S. 650.

1046 Scheffler, Wolfram: Besteuerung von Unternehmen, S. 245.

1047 Bundesministerium der Finanzen (Hrsg.): Brühler Empfehlungen zur Reform der Unternehmensbesteuerung. Bericht der Kommission zur Reform der Unternehmensbesteuerung (BMF-Schriftenreihe 66), Berlin 1999, S. $11 \mathrm{ff}$.

1048 Gesetz zur Senkung der Steuersätze und zur Reform der Unternehmensbesteuerung vom 23.10.2000, BGBl. 2000 I, S. 1433.

1049 Durch den Systemwechsel wurde die bisherige Benachteiligung von Ausländern beseitigt.

1050 Für einbehaltene Gewinne ergab sich unter Berücksichtigung von Gewerbesteuer (bei einem Hebesatz von $400 \%$ ) und Solidaritätszuschlag ein Teilsteuersatz von rund $38 \%$, vgl. Hundsdoerfer, Jochen: Halbeinkünfteverfahren und Lock-In-Effekt, in: Steuer und Wirtschaft 78 (2001), S. 113-125, S. 113. 
bis zum 31. Dezember 2008 nur zur Hälfte als Einnahmen aus Kapitalvermögen anzusetzen („Halbeinkünfteverfahren“). ${ }^{1051}$

Für die im Betriebsvermögen gehaltenen Beteiligungen gilt seit dem 1. Januar $2009^{1052}$ weiterhin ein Teileinkünfteverfahren. Die Körperschaftsteuervorbelastung wurde auf 15 Prozent reduziert, und der steuerfreie Anteil des Beteiligungsertrages beträgt seitdem nur noch 40 Prozent. Aufwendungen, die in wirtschaftlichem Zusammenhang mit dem Beteiligungsertrag stehen, dürfen von der Bemessungsgrundlage zu 60 Prozent abgezogen werden. Die Erträge aus im Privatvermögen gehaltenen Beteiligungen unterliegen seit dem 1. Januar 2009 der Abgeltungsteuer in Höhe von 25 Prozent. ${ }^{1053}$ Der Steuerpflichtige kann eine Veranlagung beantragen, wenn sein persönlicher Grenzsteuersatz unter 25 Prozent liegt. Werbungskosten, die im Zusammenhang mit dem Beteiligungsertrag stehen, können nicht über den Sparer-Pauschbetrag hinaus geltend gemacht werden. ${ }^{1054}$

Sofern eine Körperschaft selbst Anteilseignerin einer anderen Körperschaft ist, bleiben die von der Tochterkörperschaft ausgeschütteten Dividenden bei ihr grundsätzlich steuerfrei. Für den Erlös aus der Veräußerung von Beteiligungen an Körperschaften gilt für natürliche Personen entweder das Teileinkünfteverfahren (Veräußerungsgewinne im Sinn des $\$ 17$ EStG sowie im Betriebsvermögen gehaltene Beteiligungen) oder die Abgeltungsteuer (im Privatvermögen gehaltene Beteiligungen, wenn diese unter 1 Prozent liegen).

\subsubsection{Kritische Würdigung}

Seitdem sich der Gesetzgeber mit dem Körperschaftsteuergesetz 1920 für den Dualismus der Unternehmensbesteuerung entschieden hatte, ist das Unternehmensteuerrecht in seinen Grundstrukturen unverändert geblieben. Mit dem Dualismus von Körperschaft- und Einkommensteuer ist das Problem der Doppelbelastung jedoch untrennbar verbunden. Nach dem Grundsatz der Gleichmäßigkeit der Besteuerung sollte jedoch eine Wahl zwischen verschiedenen Rechtsformen nicht verzerrt wer-

1051 Hey, Johanna: $\$ 11$ Körperschaftsteuer, S. 673 f.

1052 Unternehmensteuerreformgesetz 2008 vom 14.08.2007, BGBl. I 2007, S. 1912.

1053 Bereits im Jahr 1952 wurde bei den Einkünften aus Kapitalvermögen die progressive Einkommensteuer durch eine Kapitalertragsteuer von 30 \% ersetzt; vgl. das Gesetz zur Förderung des Kapitalmarkts vom 15.12.1952, BGBl. I 1952, S. 793.

1054 Es sei denn, es handelt sich um eine typischerweise unternehmerische Beteiligung, für die $\$ 32 \mathrm{~d}$ II Nr. 3 EStG die Veranlagung im Teileinkünfteverfahren i. V. mit $\$ 3 \mathrm{c}$ II EStG vorsieht; vgl. Hey, Johanna: $\$ 11$ Körperschaftsteuer, S. 674. 
den, denn Rechtsformneutralität ist eine Ausprägung des Gebots gleichmäßiger Besteuerung nach der wirtschaftlichen Leistungsfähigkeit. ${ }^{1055}$

Rechtsformneutralität der Besteuerung ist beim klassischen Körperschaftsteuersystem nicht gegeben, da bei diesem ausgeschüttete Gewinne einer Doppelbelastung unterliegen. Neben die Körperschaftsteuer der Kapitalgesellschaft tritt die ungeschmälerte Besteuerung der Dividende beim Anteilseigner mit der Einkommensteuer. Darüberhinaus ist ein klassisches Körperschaftsteuersystem wegen der Mehrbelastung von Kapitalgesellschaften nicht rechtsformneutral, wegen der Benachteiligung der Eigenfinanzierung nicht finanzierungsneutral und wegen der Bevorzugung der Gewinnthesaurierung nicht gewinnverwendungsneutral. ${ }^{1056}$

Demgegenüber vermeidet das körperschaftsteuerliche Anrechnungsverfahren vollständig eine Doppelbelastung von Gewinnen, die eine Kapitalgesellschaft ausschüttet. ${ }^{1057}$ Zwar liegen die Vorteile des körperschaftlichen Anrechnungsverfahrens bei rein nationalen Sachverhalten darin, dass es rechtsformneutral und finanzierungsneutral ist und in seiner Konzeption keine Tendenz zur Thesaurierung von Gewinnen auslöst. Jedoch ist dieses Verfahren nicht „europatauglich“, da grenzüberschreitende Sachverhalte benachteiligt werden, worin ein Verstoß gegen die Kapitalverkehrsfreiheit gesehen wird. Aus diesem Grund wurde das körperschaftsteuerliche Anrechnungsverfahren im Jahr 2000 in Deutschland aufgegeben. ${ }^{1058}$

Bei dem in Deutschland geltenden Körperschaftsteuersystem zur Besteuerung von Dividenden handelt es sich um eine spezifische Form der Körperschaftsteuersysteme, bei denen die Doppelbelastung in pauschalierter Form gemildert wird. Die Milderung der Doppelbesteuerung erfolgt dadurch, dass die Gewinnausschüttungen auf der Ebene des Anteilseigners einer begünstigten Besteuerung unterliegen (sogenanntes Shareholder-Relief-System). ${ }^{1059}$ Die Nachteile dieses geltenden Körper-

1055 Hey, Johanna: $\$ 13$ Rechtsformneutralität der Unternehmensbesteuerung, in: Tipke, Klaus/Lang, Joachim (Hrsg.): Steuerrecht, fortgeführt von Roman Seer u. a., 22. Aufl., Köln 2015, S. 777-783. Auf die Rechtsformunterschiede zwischen Personenunternehmen und Kapitalgesellschaften in der Erbschaft- und Schenkungsteuer wird hier nicht eingegangen.

1056 Scheffler, Wolfram: Besteuerung von Unternehmen, S. 243.

1057 Wie beim körperschaftsteuerlichen Anrechnungsverfahren wird sowohl beim Dividendenabzugsverfahren als auch beim Dividendenfreistellungsverfahren eine Doppelbelastung der von einer Kapitalgesellschaft ausgeschütteten Gewinne in vollem Umfang vermieden. Sie sind jedoch im grenzüberschreitenden Bereich mit erheblichen Nachteilen verbunden und werden in Deutschland nicht angewendet. Vgl. hierzu Scheffler, Wolfram: Besteuerung von Unternehmen, S. 247-250, S. 234.

1058 Scheffler, Wolfram: Besteuerung von Unternehmen, S. 245.

1059 Ebd., S. 233. 
schaftsteuersystems sind fehlende Rechtsform-, fehlende Finanzierungs-, fehlende Gewinnverwendungsneutralität ${ }^{1060}$, und es ist mit dem Prinzip der Gleichmäßigkeit der Besteuerung unvereinbar.

Mit dem Systemwechsel ab dem Jahr 2001 wurden die tariflichen Unternehmensteuersätze abgesenkt. Die daraus folgenden Einnahmeausfälle wurden zu einem Teil durch Einschränkungen bei der steuerlichen Gewinnermittlung finanziert. ${ }^{1061}$ Zum anderen wurden durch das Unternehmensteuerreformgesetz 2008 ertragsunabhängige Elemente zur Besteuerung herangezogen, indem der Abzug betrieblicher Aufwendungen teilweise oder vollständig versagt wurde und damit der Gewinn keine reine Nettogröße mehr darstellte. ${ }^{1062}$

Die Einschnitte in das objektive Nettoprinzip stellen einen steuersystematischen Rückschritt dar. So erfolgte die Verbreiterung der Bemessungsgrundlage u. a. durch Einschränkungen von Verlustvorträgen und durch die Einführung der sogenannten Zinsschranke. Die konkrete Ausgestaltung der Abgeltungsteuer ist außerdem mit dem Prinzip einer gleichmäßigen Besteuerung nicht vereinbar. ${ }^{1063}$

1060 Die Besteuerung von ausgeschütteten Gewinnen hat Einfluss auf die Rechtsformwahl, denn sie hängt davon ab, in welcher Rechtsform das Unternehmen geführt wird. Der Körperschaftsteuer wird der Charakter einer eigenständigen Unternehmensteuer zugewiesen, die auch im Ausschüttungsfall bestehen bleibt. Bei Einzelunternehmen und Personengesellschaften bestimmt sich die Steuerbelastung nach dem persönlichen Einkommensteuersatz des Inhabers bzw. Anteilseigners. Auch Finanzierungsentscheidungen werden beeinflusst, denn ebenso wie bei der Rechtsformwahl hängt dies davon ab, ob der Gesellschafter die Kapitalgesellschaft mit Eigenkapital oder mit Fremdkapital finanzieren soll. Unabhängig davon wird das Gebot der Finanzierungsneutralität verletzt, weil Dividenden im Gegensatz zu nicht vorbelasteten Zinseinkünften steuerlich benachteiligt werden. Die Ausschüttung von Gewinnen gegenüber der Einbehaltung von Gewinnen ist generell mit einer Mehrbelastung verbunden, was eine Tendenz zur Gewinnthesaurierung auslöst. Vgl. hierzu im Einzelnen Scheffler, Wolfram: Besteuerung von Unternehmen, S. 238-241; Hey, Johanna: \$11 Körperschaftsteuer, S. 676-678.

1061 Spengel, Christoph/Zinn, Benedikt: Konsequenzen und Folgerungen aus den Unternehmenssteuerreformen in Deutschland in den vergangenen 20 Jahren, in: Tipke, Klaus u. a. (Hrsg.): Gestaltung der Steuerrechtsordnung. Festschrift für Joachim Lang zum 70. Geburtstag, Köln 2010, S. 399-422, S. 399.

1062 Ebd., S. 399 f.

1063 Spengel, Christoph/Zinn, Benedikt: Konsequenzen und Folgerungen, S. 400-416; Hey, Johanna: Verletzung fundamentaler Besteuerungsprinzipien durch die Gegenfinanzierungsmaßnahmen des Unternehmensteuerreformgesetzes 2008, in: Betriebs-Berater 62/24 (2007), S. 1303-1309; Seer, Roman: Personenunternehmerbesteuerung - Zur Willkürlichkeit des Einkunftsarten-Steuerrechts, in: Tipke, Klaus u. a. (Hrsg.): Gestaltung der Steuerrechtsordnung. Festschrift für Joachim Lang zum 70. Geburtstag, Köln 2010, S.655-681, S. 664f.; Drüen, Klaus-Dieter: Die Bruttobesteuerung, S. 4. 
Das Problem der Doppelbelastung wurde zwar aufgrund der Absenkung der Vorbelastung durch die Steuerreform 2008 vermindert, eliminierte es aber nicht. Auch bei der Bewertung der Belastungswirkungen beim Anteilseigner zeigen sich Verzerrungen der Belastungsrelation, je nachdem ob die Beteiligung im Betriebsoder Privatvermögen gehalten wird.

Allerdings ist dieses Shareholder-Relief-System „europatauglich“, was das Hauptargument für die Einführung dieses Systems in Deutschland durch die Unternehmensteuerreform im Jahr 2000 war. Bei diesem System stehen der Bundesrepublik Deutschland sowohl bei Beteiligungen von inländischen Anteilseignern an ausländischen Kapitalgesellschaften ${ }^{1064}$ als auch bei Beteiligungen von ausländischen Anteilseignern an inländischen Kapitalgesellschaften ${ }^{1065}$ ein Besteuerungsrecht zu. Es kommt demgemäß nicht zu einer einseitigen Aufteilung der Besteuerungsrechte. ${ }^{1066}$

\subsubsection{Methoden zur Verwirklichung der Rechtsformneutralität}

Nach dem Grundsatz der Gleichmäßigkeit der Besteuerung soll eine Wahl zwischen verschiedenen Rechtsformen nicht verzerrt werden, denn nur ein systemgerechtes und folgerichtig ausgestaltetes Unternehmensbesteuerungssystem kann gerecht sein. Der durch die Globalisierung der Weltwirtschaft erheblich verschärfte Steuerwettbewerb zwingt den Gesetzgeber, die Wettbewerbsfähigkeit von Deutschland zu stärken. Dabei kommt Steuern, insbesondere Steuersätzen eine Signalwirkung im Staatenwettbewerb zu. Ebenso bedeutend wie eine niedrige Tarifbelastung der Unternehmen ist ein nicht streitanfälliges Steuerrecht, das wirtschaftliche Entscheidungen wenig verzerrt, Steuerplanungssicherheit bietet und eine störungsfreie Zusammenarbeit mit den Finanzbehörden gewährleistet. ${ }^{1067}$

Von der Wissenschaft sind in den vergangenen Jahrzehnten eine Vielzahl von Methoden zur Rechtsformneutralität der Unternehmensbesteuerung entwickelt worden mit dem Ziel, das Gebot gleichmäßiger Besteuerung nach der wirtschaftlichen Leistungsfähigkeit zu erreichen. Da die Hauptursache für die unterschiedlichen Belastungsdifferenzen zwischen Kapitalgesellschaften und Einzelunternehmen

1064 Sogenannter Outboundfall, eine Doppelbelastung wird in pauschalierter Form vermieden; vgl. im Einzelnen Scheffler, Wolfram: Besteuerung von Unternehmen, S. 245-248.

1065 Sogenannter Inboundfall, bei dem ebenfalls eine Doppelbelastung vermieden wird; vgl. im Einzelnen ebd., S. 245-248.

1066 Scheffler, Wolfram: Besteuerung von Unternehmen, S. 242. Bemerkenswert ist, dass die Mehrzahl der EU-Länder ein mit der deutschen Abgeltungsteuer sowie dem deutschen Teileinkünfteverfahren vergleichbares Körperschaftsteuersystem haben und auch international eine Tendenz zu Körperschaftsteuersystemen besteht, die die Doppelbelastung in pauschalierter Form vermeiden, ebd., S. 250.

1067 Hey, Johanna: $\$ 7$ Einführung in das besondere Steuerschuldrecht, S. 268. 
bzw. Personengesellschaften das duale System bildet, also das Nebeneinander von natürlichen und juristischen Personen als Steuersubjekte, wird zunächst auf die Konzepte zur Aufgabe des dualen Systems eingegangen. ${ }^{1068}$

\subsection{Reformvorschläge bei Aufgabe des dualen Systems}

Legt man bei einer einheitlichen Gesamtkonzeption der Besteuerung von Einzelunternehmen als Vorstellung zugrunde, dass bei allen Unternehmen nur die hinter den Unternehmen stehenden Personen besteuert werden, führt dies zu einer Teilhabersteuer. Wird dagegen das Unternehmen - wie bei einer Kapitalgesellschaft - losgelöst von den natürlichen Personen betrachtet, führt dies zur Betriebsteuer.

\section{Teilhabersteuer}

Nach der ökonomischen Forderung nach Rechtsformneutralität sollen auch Kapitalgesellschaften transparent besteuert werden, indem thesaurierte oder ausgeschüttete Gewinne dem Anteilseigner unmittelbar zugerechnet und nur der Einkommensteuer unterworfen werden. ${ }^{1069}$

\section{Betriebsteuer / Grundmodell}

Bei der Betriebsteuer ist die zivilrechtliche Rechtsfähigkeit des Unternehmens nicht entscheidend, sondern jedes Unternehmen - sei es eine Kapitalgesellschaft, ein Einzelunternehmen oder eine Personengesellschaft - bildet selbstständig das Anknüpfungsmerkmal für die Besteuerung. Die Steuerbelastung des Unternehmers bzw. Gesellschafters wird durch die Erfolge des Unternehmens, unabhängig davon, ob die Gewinne thesauriert oder ausgeschüttet werden oder Verluste entstehen, nicht beeinflusst. Durch eine Betriebsteuer soll ermöglicht werden, dass alle Unternehmen nach dem gleichen Konzept besteuert werden und damit die Höhe der Steuerbelastung nicht von der gewählten Rechtsform beeinflusst wird. ${ }^{1070}$

\section{Modifizierte Betriebsteuer}

Aufgrund der Kritik der ungleichmäßigen Behandlung gewerblicher und nichtgewerblicher Einkünfte wurde das Konzept der modifizierten Betriebsteuer entwickelt. Jeder gewerbliche, freiberufliche, land- und forstwirtschaftliche Betrieb sowie die

1068 Jacobs, Otto H./Scheffler, Wolfram/Spengel, Christoph: Unternehmensbesteuerung und Rechtsform, S. 108-139.

1069 Hey, Johanna: $\$ 13$ Rechtsformneutralität der Unternehmensbesteuerung, S. 780.

1070 Jacobs, Otto H./Scheffler, Wolfram/Spengel, Christoph: Unternehmensbesteuerung und Rechtsform, S. $111 \mathrm{f}$. 
private Vermögensverwaltung ist ein Betrieb im Sinne dieses Konzepts. Durch die Erweiterung des Anwendungsbereiches soll erreicht werden, dass der Betriebsteuer die Erträge sämtlicher Finanz- und Realinvestitionen dem niedrigeren proportionalen Steuersatz unterliegen. Alle Ausschüttungen bzw. Entnahmen unterliegen einer Belastung mit der progressiven Einkommensteuer, wobei zur Vermeidung von Doppelbelastungen ein Anrechnungsverfahren vorgesehen ist. ${ }^{1071}$

\section{Bundessteuergesetzbuch von Paul Kirchhof}

Das von Paul Kirchhof im Jahr 2011 vorgelegte Bundesteuergesetzbuch ${ }^{1072}$ beeinhaltet die Vorschriften zur Ertragsbesteuerung von natürlichen und juristischen Personen, das Umsatzsteuergesetz, das Erbschaft- und Schenkungsteuergesetz sowie das allgemeine Verbrauchsteuergesetz. Kernelement dieser Reform der Einkommenund Unternehmensbesteuerung ist die Einführung einer sogenannten Flat Rate, die bewirken soll, alle Arten von Einkommen einem einheitlichen, vergleichsweise niedrigen proportionalen Steuersatz zu unterwerfen. Mit diesem Konzept sollen das Steuerrecht vereinfacht, die Gleichmäßigkeit der Besteuerung sichergestellt und die Lenkungs- und Subventionsnormen zurückgeführt werden. ${ }^{1073}$

\section{„Dual Income Tax“ / Das Nordische Modell}

Nach dem Nordischen Modell erfolgt im Rahmen einer „Dual Income Tax“ eine strikte Trennung zwischen Kapitaleinkommen (capital income) und Arbeitseinkommen (earned income). Am konsequentesten wurde die gesonderte Besteuerung von Kapitaleinkünften in Finnland, Schweden und Norwegen durchgeführt. In Deutschland wurden zwei weitere Modelle mit jeweils unterschiedlichen Anknüpfungspunkten vorgestellt, auf die im Folgenden kurz eingegangen werden soll. ${ }^{1074}$

1071 Ebd., S. 112-115. Vergleichbar mit der modifizierten Betriebsteuer ist das von Joachim Lang entworfene Modell einer Inhabersteuer; vgl. Lang, Joachim: Perspektiven der Unternehmensteuerreform, in: Bundesministerium der Finanzen (Hrsg.): Brühler Empfehlungen zur Reform der Unternehmensbesteuerung. Bericht der Kommission zur Reform der Unternehmensbesteuerung (BMF-Schriftenreihe 66), Berlin 1999, Anhang Nr. 1, S. 19 ff. Zu den Brühler Empfehlungen vgl. die Ausführungen in Kapitel 10.1.2.2.2.

1072 Kirchhof, Paul: Bundessteuergesetzbuch. Ein Reformentwurf zur Erneuerung des Steuerrechts, Heidelberg 2011.

1073 Jacobs, Otto H./Scheffler, Wolfram/Spengel, Christoph: Unternehmensbesteuerung und Rechtsform, S. 115.

1074 Ebd., S. 120-128. 


\section{Die Einheitliche Unternehmensteuer der Kommission "Steuergesetzbuch" der Stiftung Marktwirtschaft}

Die Unternehmensteuerreform der Stiftung Marktwirtschaft hat eine Einheitliche Unternehmensteuer zum Ziel, welche die steuerliche Gleichbehandlung von Personenunternehmen und Einzelunternehmen neben Kapitalgesellschaften erlaubt. Die Unternehmensbelastung soll auf ein international wettbewerbsfähiges Niveau gesenkt werden. Damit können auch Gesellschafter einer Personengesellschaft und Einzelunternehmer von einer niedrigeren Besteuerung einbehaltener Gewinne profitieren. Als zusätzliches Element beeinhaltet die Einheitliche Unternehmensteuer die Kommunale Unternehmensteuer als Ersatz für die Gewerbesteuer. Bei Ausschüttung bzw. Entnahme der Gewinne aus einem Unternehmen sollen diese unter Berücksichtigung der Vorbelastung nachbelastet werden, sodass diese Kombination maximal zu einer dem Einkommensteuerspitzensatz entsprechenden Besteuerung führt.

Ziel der Kommisssion „Steuergesetzbuch“ der Stiftung Marktwirtschaft war es auch, die lenkungs- und verteilungspolitischen Maßnahmen aus dem Steuerrecht herauszunehmen und die Steuerlasten gleichmäßig allen Bürgern nach Maßgabe ihrer wirtschaftlichen Leistungsfähigkeit zuzuweisen. ${ }^{1075}$

\section{Die Duale Einkommensteuer von SVR, MPI und ZEW}

Der Sachverständigenrat zur Begutachtung der gesamtwirtschaftlichen Entwicklung (SVR) hat in Gemeinschaft mit dem Max-Planck-Institut für Geistiges Eigentum, Wettbewerbs- und Steuerrecht (MPI) und dem Zentrum für Europäische Wirtschaftsforschung (ZEW) im Jahr 2006 eine Expertise erarbeitet, in der sich die teilnehmenden Partner für ein duales System von Kapitaleinkommen einerseits und

1075 Eilfort, Michael/Lang, Joachim: Steuerpolitisches Programm, in: Lang, Joachim/ Eilfort, Michael (Hrsg.): Strukturreform der deutschen Ertragsteuern. Bericht über die Arbeit und Entwürfe der Kommission „Steuergesetzbuch“ der Stiftung Marktwirtschaft, München 2013, S. 51-110, S. 52 ff.; Jacobs, Otto H./Scheffler, Wolfram/Spengel, Christoph: Unternehmensbesteuerung und Rechtsform, S. $125 \mathrm{f}$. Dieses Reformkonzept entspricht von seinem Ansatz einer dualen Einkommensteuer, da die im Unternehmen erwirtschafteten und ausgeschütteten Gewinne auf Ebene des Unternehmens einem anderen Steuersatz unterliegen als die nichtunternehmerischen Einkünfte einer natürlichen Person; vgl. Sachverständigenrat zur Begutachtung der gesamtwirtschaftlichen Entwicklung (SVR)/Max-Planck-Institut für Geistiges Eigentum, Wettbewerbs- und Steuerrecht (MPI)/Zentrum für Europäische Wirtschaftsforschung GmbH (ZEW): Reform der Einkommens- und Unternehmensbesteuerung durch die Duale Einkommensteuer. Expertise im Auftrag der Bundesminister der Finanzen und für Wirtschaft und Arbeit vom 23. Februar 2005, Wiesbaden 2006, S. 1-321, S. 11-17. 
Arbeitseinkommen andererseits ausgesprochen haben. ${ }^{1076}$ Die Kapitaleinkommen, wie z. B. Gewinne, Ausschüttungen und Zinsen unterliegen einem einheitlichen, ermäßigten Steuersatz von 25 Prozent, unabhängig davon, ob sie innerhalb oder außerhalb eines Unternehmens erzielt werden. Alle übrigen Einkommen (Erwerbseinkommen) werden nach einem progressiven Einkommensteuertarif mit einem Spitzensteuersatz von 42 Prozent zuzüglich Solidaritätszuschlag besteuert. Diese Regelungen sollen unabhängig von der Rechtsform des Unternehmens gelten, die Gewerbesteuer soll abgeschafft bzw. als Zuschlag zur Einkommensteuer bzw. Körperschaftsteuer erhoben werden. ${ }^{1077}$

\section{Konsumorientierte Steuersysteme}

Anknüpfungspunkt der konsumorientierten Besteuerung ist, dass die wirtschaftliche Leistungsfähigkeit nicht am verwirklichten (Markt-)Einkommen, sondern konsequent am Lebenskonsum gemessen wird. Der Übergang auf eine konsumorientierte Besteuerung hat sowohl auf Unternehmensebene als auch auf Ebene der privaten Investoren bzw. Haushaltungen Konsequenzen für das Körperschaftsteuersystem, die Einkommensteuer und die Körperschaftsteuersätze sowie die anderen laufenden Steuerarten bis hin zur Gewichtung von periodischen und aperiodischen Besteuerungstatbeständen. In der Literatur werden zur Rechtsformneutralität verschiedene Grundformen vorgeschlagen, die die Anforderungen an eine wettbewerbsneutrale Besteuerung herstellen sollen. ${ }^{1078}$

\subsection{3.2 Reformvorschläge bei Beibehaltung des dualen Systems}

Mit der Einführung des Körperschaftsteuergesetzes 1920 war allein die zivilrechtliche Form der Besteuerung maßgeblich. Seitdem sind Gesellschaften und Anteilseigner zwei voneinander selbstständige Rechtssubjekte mit der Konsequenz einer strikten

1076 Sachverständigenrat zur Begutachtung der gesamtwirtschaftlichen Entwicklung (SVR)/Max-Planck-Institut für Geistiges Eigentum, Wettbewerbs- und Steuerrecht (MPI)/Zentrum für Europäische Wirtschaftsforschung GmbH (ZEW): Reform der Einkommens- und Unternehmensbesteuerung, S. 1-321.

1077 Jacobs, Otto H./Scheffler, Wolfram/Spengel, Christoph: Unternehmensbesteuerung und Rechts-form, S. 127.

1078 Ebd., S. 129-139. Verschiedene Reformmodelle zur Besteuerung des für konsumtive Zwecke verwendbaren Einkommens werden u.a. vorgeschlagen von Rose, Manfred: Vom Steuerchaos zur Einfachsteuer. Der Wegweiser durch die Steuerdebatte, Stuttgart 2003; Mitschke, Joachim: Erneuerung des deutschen Einkommensteuerrechts. Gesetzestextentwurf und Begründung. Mit einer Grundsicherungsvariante, Köln 2004; Elicker, Michael: Entwurf einer proportionalen Netto-Einkommensteuer. Textentwurf und Begründung, Köln 2004. 
Trennung zwischen der Ebene der Gesellschaft und des Anteilseigners. ${ }^{1079}$ Personengesellschaften werden nach dem Transparenzprinzip besteuert, was zur Folge hat, dass der Gewinn der Gesellschafter der Einkommensteuer unterliegt. Kritiker dieses Systems schlagen vor, das duale System beizubehalten, die Abgrenzung jedoch nicht nach der zivilrechtlichen Form, sondern anhand der wirtschaftlichen Verhältnisse vorzunehmen. Bei diesen Überlegungen kann eine Abgrenzung nach den Verhältnissen auf Ebene der Gesellschaft oder nach der Betätigung des Gesellschafters vorgenommen werden.

Bei einer Abgrenzung nach den Verhältnissen auf Ebene der Gesellschaft wird zwischen personenbezogenen und kapitalistisch ausgestalteten Unternehmen differenziert. Maßgeblich ist, ob die persönliche Verbundenheit der Gesellschafter das Unternehmen dominiert - dann wird sie wie eine Personengesellschaft besteuert - oder ob diese körperperschaftlich organisiert ist - dann wird sie körperschaftsteuerpflichtig. Umgekehrt können auch personenbezogene Kapitalgesellschaften, bei denen die Geschäftsführung durch die überwiegende Zahl der Gesellschafter ausgeübt wird, wie Personengesellschaften besteuert werden.

Bei einer Abgrenzung nach der Betätigung des Gesellschafters wird auf die Verhältnisse des Einzelfalls abgestellt. Dabei verläuft die Grenzziehung in Abhängigkeit von der Stellung und Funktion jedes einzelnen Gesellschafters zwischen unternehmerisch tätigen Personen und nur kapitalmäßig beteiligten Anteilseignern. Für die unternehmerisch tätigen Gesellschafter werden die Einkünfte nach der Mitunternehmerkonzeption festgestellt. Gesellschafter, die mit ihrer Kapitalanlage lediglich Anlagezwecke verfolgen, erzielen Einkünfte aus Kapitalvermögen. ${ }^{1080}$

\subsection{Zum Grundsatz der Besteuerung nach der Leistungsfähigkeit}

\subsubsection{Verfassungsrechtliche Begründung des Leistungsfähigkeitsprinzips}

Bereits in der Erklärung der Menschen- und Bürgerrechte vom 26. August 1789 war das Leistungsfähigkeitsprinzip in Artikel 13 verankert: „Für den Unterhalt der Streitmacht und für die Kosten der Verwaltung ist eine allgemeine Abgabe

1079 Vgl. Kapital 10.1.2.2.1.

1080 Jacobs, Otto H./Scheffler, Wolfram/Spengel, Christoph: Unternehmensbesteuerung und Rechtsform, S. 139-141. 
unumgänglich. Sie muss gleichmäßig auf alle Bürger unter Berücksichtigung ihrer Vermögensumstände verteilt werden. ${ }^{\text {"1081 }}$ Die erste gesamtdeutsche Verfassung, in der das Leistungsfähigkeitsprinzip kodifiziert war, war die Weimarer Reichsverfassung vom 11. August 1919. In Art. 134 dieser Verfassung hieß es: „Alle Staatsbürger ohne Unterschied tragen im Verhältnis ihrer Mittel zu allen öffentlichen Lasten nach Maßgabe der Gesetze bei." Mit Aufnahme des Leistungsfähigkeitsprinzips in die Weimarer Reichsverfassung wurde dieser Besteuerungsgrundsatz Gegenstand der Verfassungsauslegung. Die umfassendste Untersuchung stammt von Albert Hensel, der in seinen Ausführungen zu Art. 134 WRV ausführt, dass ,es unbestritten und einhellige Meinung [ist ...], daß nur ein Progressivsteuersystem den Anforderungen an Gerechtigkeit Genüge leistet. ${ }^{\text {"1082 }}$

Im Gegensatz zur Weimarer Reichsverfassung gibt es im Grundgesetz keine direkte verfassungsrechtliche Festschreibung des Leistungsfähigkeitsprinzips. Das Bundesverfassungsgericht vertritt die Auffassung, dass das Prinzip der Besteuerung nach der wirtschaftlichen Leistungsfähigkeit indirekt aus Art. 3 Abs. 1 GG abzuleiten sei; ${ }^{1083}$ denn aus dem allgemeinen Gleichheitssatz folge das Gebot der Steuergerechtigkeit. Dieses Gebot verlange, dass die wirtschaftliche Leistungsfähigkeit bei der Bemessung der Besteuerung unbedingt zu beachten sei, um eine verhältnismäßige und gleichmäßige Steuerlastverteilung herstellen zu können. ${ }^{1084}$

Für die verfassungsrechtliche Begründung einer Besteuerung nach der wirtschaftlichen Leistungsfähigkeit wird vielfach in der Staatsrechtslehre nicht nur Art. 3 Abs. 1 GG, sondern auch der in Art. 20 Abs. 1 und Art. 28 Abs. 1 GG ausformulierte Sozialstaatsgedanke herangezogen. ${ }^{1085}$ Ebenfalls wird das Leistungsfähigkeitsprinzip aus dem in Art. 20 Abs. 3, Art. 1 Abs. 3 und Art. 19 Abs.

1081 Nach Tipke, Klaus: Die Steuerrechtsordnung, S.488, darf der französische Begriff „faculté" hier nicht mit Vermögen (fortune) übersetzt werden, sondern muss mit (steuerlicher) „Leistungsfähigkeit“ oder (steuerlichem) „Leistungsvermögen“ übertragen werden.

1082 Hensel, Albert: Verfassungsrechtliche Bindungen, S. 469; vgl. auch die Ausführungen in Kapitel 8.1.

1083 BVerfGE 55, 274, 302; 61, 319, 343 f.; 66, 214, 223; 67, 290, 297; 68, 143, 152; 74, 182, 199.

1084 Z. B. BVerfGE 8, 51, 47,1. Es ist weltweit anerkannt, dass das Leistungsfähigkeitsprinzip der grundlegende sachgerechte Wertmaßstab für den Bereich des Fiskal-Steuerrechts das Ausgangs- oder Leitprinzip sein sollte; vgl. Tipke, Klaus: Steuerrecht als Wissenschaft, S. 33 .

1085 Kirchhof, Paul: Steuergleichheit, S. 306; Tipke führt hierzu aus: „Daß das Leistungsfähigkeitsprinzip sich über die Jahrhunderte durchgesetzt hat, hängt wesentlich mit dem Durchbruch des Sozialstaatsgedankens zusammen." Tipke, Klaus: Die Steuerrechtsordnung, S. 480 . 
4 GG und in den Grundrechten verankerten Rechtsstaatsprinzip ${ }^{1086}$ sowie dem verfassungsrechtlichen Schutz von Ehe und Familie nach Art. 6 Abs. 1 GG und aus bestimmten Freiheitsgrundrechten, insbesondere Art. 12 Abs. 1, Art. 14 Abs. 1 und Art. 2 Abs. 1 GG, interpretiert. ${ }^{1087}$

\subsubsection{Zulässigkeit progressiver Besteuerung}

Das Bundesverfassungsgericht leitet den progressiven Einkommensteuertarif unmittelbar und allein aus dem Gleichheitssatz ab. ${ }^{1088}$ In der Literatur hingegen wird die progressive Steuer als ein zulässiges und mögliches, nicht als zwingendes Mittel der Verwirklichung sozialer Gerechtigkeit angesehen. ${ }^{1089}$ Joachim Lang führt hierzu aus:

Die Gerechtigkeit des progressiven Einkommensteuertarifs, daß der wirtschaftlich Leistungsfähigere einen höheren Prozentsatz seines Einkommens als Steuer zu zahlen hat als der wirtschaftlich Schwächere, ist Ausdruck einer sozialstaatlich relativierten Gleichheit. ${ }^{1090}$

Ein bestimmter Progressionsverlauf lässt sich steuerwissenschaftlich nicht ableiten; es liegt im Ermessen des Gesetzgebers den Grad der Progression festzulegen. ${ }^{1091}$

1086 Davidsohn führt hierzu aus: „Eine unverhältnismäßige, der persönlichen Leistungsfähigkeit nicht entsprechende Besteuerung dürfte insbesondere auch einen Verstoß gegen das Rechtsstaatsprinzip darstellen, weil dieses Prinzip den Grundsatz der Verhältnismäßigkeit und das Übermaßverbot als verfassungsrechtliche Schranken mit umfasst.“ Davidsohn, Lars: Verfassungsrechtliche Würdigung, S. 52.

1087 Ebd., S. 51.

1088 BVerfGE 8, S. 51 und S. 68 f.

1089 Tipke, Klaus: Die Steuerrechtsordnung, S. 404; auch Kirchhof ist der Meinung, dass die Einkommensteuerprogression sozialstaatlich gerechtfertigt sei; Kirchhof, Paul: Einkommensteuergesetz. Kompaktkommentar, in: Ders. u.a. (Hrsg.): Einkommensteuergesetz mit Aktualisierungsservice, Loseblattsammlung, Heidelberg Stand: 2000, $\$ 2$ RNr. A 602.

1090 Lang, Joachim: Die Bemessungsgrundlage der Einkommensteuer. Rechtssystematische Grundlagen steuerlicher Leistungsfähigkeit im deutschen Einkommensteuerrecht, Köln 1988, S. 165, Anm. 522.

1091 Tipke, Klaus: Die Steuerrechtsordnung, S. 405. 


\subsubsection{Die Berücksichtigung der persönlichen Lebensbedingungen}

Nationalökonomen erkannten insbesondere im 19. Jahrhundert, dass gleiches Einkommen nicht unbedingt eine gleiche Steuerfähigkeit zur Folge hat. ${ }^{1092}$ Das Erwerbseinkommen dürfe nicht mit steuerlicher Leistungsfähigkeit gleichgesetzt werden, da die persönlichen und sozialen die Steuerfähigkeit verstärkenden und abschwächenden Momente bei der Veranlagung einer Steuer zu berücksichtigen sind.

Das Bundesverfassungsgericht leitet das Gebot der Steuerfreiheit des Existenzminimums in ständiger Rechtsprechung aus Art. 1 GG in Verbindung mit dem Sozialstaatsprinzip des Art. 20, Abs. 1, Art. 3, Abs. 1 und Art. 6, Abs. 1 GG sowie den Freiheitsrechten ab. Für das steuerrechtliche Existenzminimum als verfassungsrechtlich bestimmte Untergrenze gilt das sozialhilferechtlich festgelegte Leistungsniveau. ${ }^{1093}$

Ehe und Familie stehen nach Art. 6, Abs. 1 GG unter dem besonderen Schutz des Staates. Nach dem sogenannten subjektiven Nettoprinzip müssen die für das Existenzminimum des Steuerpflichtigen und seiner Familie benötigten Aufwendungen in Höhe der staatlichen Existenzsicherung durch Sozialleistungen steuerfrei gestellt sein. Ebenfalls müssen nach dem Prinzip familiärer Einkommensverteilung zwangsläufige Unterhaltsleistungen realitätsgerecht berücksichtigt werden. ${ }^{1094}$

1092 Wagner, Adolph: Finanzwissenschaft, 2. Teil, S. 446 f.; Neumann, Friedrich Julius: Die progressive Einkommensteuer, S. 176f.; Mann, Fritz Karl: Steuerpolitische Ideale, S. 306.

1093 Ständige Rechtsprechung seit BVerfGE 82, 60 (85f.); Hey, Johanna: $\$ 3$ Steuersystem und Steuerverfassungsrecht, S. $105 \mathrm{f}$. Zum Verhältnis der steuerlichen Behandlung des Existenzminimums, zum Postulat der Steuergerechtigkeit, zum Einkommensbegriff, zur Verteilungsfunktion der Besteuerung und zu den ökonomischen Güte- und Qualitätseigenschaften des Steuersystems vgl. Treisch, Corinna: Existenzminimum und Einkommensbesteuerung. Zum Existenzminimum und Familienlastenausgleich vgl. Schneider, Dieter/Siegel, Theodor: Existenzminimum und Familienlastenausgleich: Ein Problem der Reform des Einkommensteuerrechts, in: Deutsches Steuerrecht 17 (1994), S. 597-604.

1094 Hey, Johanna: $\$ 3$ Steuersystem und Steuerverfassungsrecht, S. 110 und S. 307-321. 


\subsubsection{Zum Grundsatz der Besteuerung nach der Leistungsfähigkeit am Beispiel der Ehegattenbesteuerung}

Das Grundgesetz enthält keine konkrete Form der Ehegattenbesteuerung. Bereits in den 1950er-Jahren hat das Bundesverfassungsgericht durch Beschluss vom 17. Januar $1957^{1095} \$ 26$ EStG in der Fassung vom 17. Januar $1952^{1096}$ „für mit Art. 6 Abs. 1 GG unvereinbar und nichtig“ erklärt, „weil Ehegatten durch die Zusammenveranlagung angesichts des auf die Leistungsfähigkeit des Einzelnen hin angelegten progressiven Steuertarifs im wirtschaftlichen Ergebnis schlechter gestellt würden als andere Personen. ${ }^{\text {"1097 }}$

Da nach diesem Beschluss eine Regelung, aus der sich Mehrsteuern bisher lediger Personen durch ihre Verheiratung ergeben, als Verstoß gegen Art. 6 Abs. 1 GG (Schutz der Ehe) angesehen wurde, hat der Gesetzgeber eine Neuordnung der Ehegattenbesteuerung herbeigeführt. In der Begründung zu dieser Neuordnung wird ausgeführt, dass auch eine Zusammenveranlagung bei einem Progressionstarif unter Anwendung des amerikanischen Splittingverfahrens (Halbierung des Gesamteinkommens der Eheleute) den Forderungen des Bundesverfassungsgerichts entspricht: Das Einkommen von Mann und Frau wird zusammengerechnet und zum Zwecke der Steuerermittlung durch zwei geteilt. Der aufgrund des Steuertarifs festgestellte Steuerbetrag wird verdoppelt und ergibt so die Steuerschuld des Ehepaares. Bei diesem Verfahren werden Mann und Frau wie zwei Ledige behandelt, die Erwerb und Verbrauch gemeinsam durchführen. ${ }^{1098}$

Die Bundesregierung hatte gegen die Einführung des Splittings zunächst Bedenken, da die Aufteilung des gemeinsamen Einkommens der Ehegatten unvermeidlich zu einem weit größeren Vorteil bei höheren als bei kleineren Einkommen führe, und wollte die Wirkung des Splitting dadurch begrenzen, dass der Unterschied in der Besteuerung des Ehepaares und des Ledigen auf einen bestimmten Höchstbetrag begrenzt wird. Im Hinblick darauf, dass dies in zahlreichen Fällen gegen die Grundsätze des Bundesverfassungsgerichtes verstoßen würde, hat die Bundesregierung „es vorgezogen, den neuen Einkommensteuertarif durch eine schnellere Progression so zu gestalten, daß sich die Vorteile gegenüber der jetzigen Besteuerung auch bei

1095 BVerfG 1 BvL 4/54; BVerfG 6,55.

1096 EStG 1951 - BGBl I, S. 33.

1097 BVerfG, Beschluss des Zweiten Senats vom 07.05.2013 - 2BvR 909/06 Rn3.

1098 BT-Drucks. 3/260 vom 07.03.1958, S. 33. 
Ehepaaren in höheren Einkommenstufen in Grenzen halten. " ${ }^{1099}$ Bis heute ist das Ehegattensplitting lebhaft umstritten. ${ }^{1100}$

\subsubsection{Das Ehegattensplitting aus Sicht der Steuerwissenschaften}

Nicht nur in der politischen Parteienlandschaft, auch in den Steuerwissenschaften wird das Ehegattensplitting ${ }^{1101}$ seit Langem kontrovers diskutiert. ${ }^{1102}$ Seit dem Beschluss des BVerfG vom 7. Mai $2013^{1103}$, das Ehegattensplitting auch auf eingetragene Lebenspartnerschaften anzuwenden, ist dieses Institut wieder verstärkt in den Blickpunkt der politischen und wissenschaftlichen Diskussion gerückt. ${ }^{1104}$

\section{Kriterien einer gerechten Ehegattenbesteuerung}

Es stellt sich die Frage, welche alternativen Besteuerungsformen für Ehegatten zur Anwendung kommen können.

Grundsätzlich kann man vier Methoden der Ehegattenbesteuerung unterscheiden: Die Haushaltsbesteuerung ist - wie bereits ausgeführt - verfassungswidrig, da das Diskriminierungsverbot es nicht gestattet, Eheleute stärker zu besteuern als zwei Unverheiratete mit entsprechendem Einkommen. ${ }^{1105}$ Bei der "reinen“ Individualbesteuerung ${ }^{1106}$ bleibt die Ehe unberücksichtigt, jeder Ehegatte wird

1099 Ebd., S. 34.

1100 Sandweg, Severin: Der Splittingtarif nach $\$ 32$ a Abs. 5 EStG - Relikt aus alten Zeiten oder ausgewogene Berücksichtigung der steuerlichen Leistungsfähigkeit?, in: Deutsches Steuerrecht 43 (2014), S. 2097-2102, S. 2097; Hey, Johanna: $\$ 3$ Steuersystem und Steuerverfassungsrecht, S. 107.

1101 Auf das Familiensplitting, das häufig nicht mit Steuergerechtigkeit, sondern mit familienpolitischen Zielen begründet wird, wird hier nicht eingegangen.

1102 Maiterth, Ralf/Chirvi, Malte: Das Ehegattensplitting aus Sicht der Steuerwissenschaften, in: Steuer und Wirtschaft 92 (2015), S. 19-32, S. 19.

1103 BVerfG vom 07.05.2013 - 2BvR 909/06 Rn3.

1104 Maiterth, Ralf/Chirvi, Malte: Das Ehegattensplitting, S. 19.

1105 Scherf, Wolfgang: Das Ehegattensplitting aus finanzwissenschaftlicher Sicht, in: Steuer und Wirtschaft 77 (2000), S. 269-278, S. 272.

1106 Mit einer kleinen Anfrage von Abgeordneten der Fraktion Bündnis 90/Die Grünen wurden die finanziellen Auswirkungen erbeten, wenn die Zusammenveranlagung von Ehegatten vollständig aufgegeben würde und das bestehende Ehegattensplitting in eine reine Individualbesteuerung umgewandelt würde. Die Bundesregierung schätzt, dass die jährlichen Steuermehreinnahmen sich auf $20 \mathrm{Mrd}$. Euro summieren würden. Vgl. Bundesregierung: Antwort auf die Kleine Anfrage [...] der Fraktion Bündnis 90/Die Grünen, Finanzielle Auswirkungen von Vorschlägen zur Reform des Ehegattensplittings und des Familienleistungsausgleichs, BT-Drucks. 17/13044 vom 11.04.2013, S. 1-4, S. 2. 
eigenständig besteuert. Dies bedeutet, dass Ledige und Verheiratete ohne Rücksicht auf ihre unterschiedlichen Lebensverhältnisse gleichbehandelt werden und der gemeinsamen Lebensführung jeder steuerliche Stellenwert abgesprochen wird. ${ }^{1107}$ Ganz unabhängig davon erscheint die Individualbesteuerung unzulässig zu sein, da das Bundesverfassungsgericht die Steuerfreistellung des familiären Existenzminimums fordert. ${ }^{1108}$ Das Ehegattentarifsplitting entspricht nach Auffassung des Bundesverfassungsgerichts

dem Grundsatz der Besteuerung nach der Leistungsfähigkeit. Es geht davon aus, dass zusammenlebende Ehegatten eine Gemeinschaft des Erwerbs- und Verbrauchs bilden, in der ein Ehegatte an den Einkünften und Lasten des anderen wirtschaftlich jeweils zur Hälfte teilhat. [...] Damit knüpft das Splitting an die wirtschaftliche Realität der intakten Durchschnittsehe an, in der ein Transfer steuerlicher Leistungsfähigkeit zwischen den Partnern stattfindet. Diese Ehegattenbesteuerung steht auch in Einklang mit den Grundwertungen des Familienrechts. Die Institute des Zugewinnausgleichs und neuerdings des Versorgungsausgleichs lassen den Grundsatz erkennen, dass das während der Ehe Erworbene gemeinschaftlich erwirtschaftet ist. ${ }^{1109}$

Das Ehegattensplitting ist danach keine Steuervergünstigung, sondern resultiert unmittelbar aus einer konsequenten Anwendung des Leistungsfähigkeitsprinzips. ${ }^{1110}$

Eine vierte Form der Ehegattenbesteuerung ist das sogenannte Ehegattenrealsplitting. ${ }^{1111}$ Diese Methode stellt eine Individualbesteuerung dar, bei der ein Betrag $\mathrm{X}$ beim besser verdienenden Ehegatten abgezogen werden kann und entsprechend beim anderen Ehegatten zu versteuern ist; eine Methode, die als „Minimalform der steuerlichen Berücksichtigung der Ehe" dem Gesetzgeber offensteht. ${ }^{112}$

1107 Scherf, Wolfgang: Das Ehegattensplitting, S. 273.

1108 Maiterth, Ralf/Chirvi, Malte: Das Ehegattensplitting, S. 21; BVerfG vom 10.11.1998, 2 BvL. 42/93.

1109 BVerfG vom 03.11.1982, 1 BvR 620/78; 1 BvR 1335/78; 1 BvR 1104/79; 1 BvR 363/80, Rz. 105.

1110 Hey, Johanna: $\$ 3$ Steuersystem und Steuerverfassungsrecht, S. 107-109.

1111 Auf die Frage, welche finanziellen Auswirkungen es hätte, wenn das vorhandene Ehegattensplitting in ein Realsplitting analog zum bestehenden Realsplitting für Geschiedene umgewandelt würde, antwortete die Bundesregierung: „Eine Individualbesteuerung von Ehepaaren mit Unterhaltsabzug bis zu 13805 Euro beim höher verdienenden Partner analog zum heutigen Realsplitting für Geschiedene würde mit jährlichen steuerlichen Mehrbelastungen von rund 3,3 Mrd. Euro einhergehen." Vgl. Bundesregierung: Antwort auf die Kleine Anfrage, S. 2.

1112 Maiterth, Ralf/Chirvi, Malte: Das Ehegattensplitting, S. $20 \mathrm{f}$. 


\section{Die Beurteilung des Ehegattensplittings im juristischen Schrifttum}

Im steuerjuristischen Schrifttum besteht weitgehende Einigkeit darüber, wie eine Besteuerung der vertraglichen Rechtsstände „Gütertrennung“ und „Gütergemeinschaft" aussehen sollte. ${ }^{1113}$ In den Fällen der Gütertrennung ist das Splitting kaum zu rechtfertigen, da keine Erwerbs- und Vermögensgemeinschaft besteht und eine Zugewinngemeinschaft nicht stattfindet. ${ }^{1114}$ Für den Güterstand der Gütergemeinschaft ist das Ehegattensplitting als gleichmäßige Besteuerung nach der Leistungsfähigkeit angemessen, da die Ehegatten das gemeinschaftliche Einkommen zivilrechtlich (\$1415 BGB) jeweils zur Hälfte in Besitz nehmen. ${ }^{1115}$

Die Beurteilung des Ehegattensplittings im steuerjuristischen Schrifttum ist äußerst umstritten, wenn es um den gesetzlichen Güterstand der Zugewinngemeinschaft geht. Befürworter sehen in Übereinstimmung mit der Auffassung des Bundesverfassungsgerichts in der Ehe eine Erwerbs- und Verbrauchsgemeinschaft. Gegner bestreiten eine derartige Existenz und verweisen darauf, dass mithin die horizontale Gleichmäßigkeit der Besteuerung verletzt wird. ${ }^{1116}$ Als bedeutsamer Kritikpunkt am Ehegattensplitting wird die Diskriminierung insbesondere von Frauen angeführt, die einen Verstoß gegen Art. 3 GG darstellen soll. Der zentrale Vorwurf lautet darin, dass die „Nur-Hausfrauentätigkeit“ gefördert würde, was in der Regel dem Ehemann zugute käme. ${ }^{117}$ Demgegenüber weist Tipke auf die familienpolitische Neutralität des Ehegattensplittings hin, denn Steuerbehörden können nicht aufklären, aus welchem Grund jemand arbeitet und ob in dieser Arbeit insbesondere ein Vorteil oder ein Nachteil gesehen wird. ${ }^{1118}$ Als weiteres bedeutsames Argument gegen das Ehegattensplitting wird angeführt, dass es die Institution Ehe, nicht jedoch Familien fördere. ${ }^{1119}$ Dabei wird unterstellt, dass immer mehr Ehepaare kinderlos seien und Kinder von Alleinerziehenden bzw. nichtverheiratenden Paaren aufgezogen werden würden. ${ }^{1120}$ Demgegenüber verweisen Löhr und Serwe darauf, dass aufgrund empirischer Untersuchungen der

1113 Ebd., S. 21.

1114 Tipke, Klaus: Die Steuerrechtsordnung, S. 379; Vogel, Klaus: Besteuerung von Eheleuten und Verfassungsrecht, in: Steuer und Wirtschaft 76 (1999), S. 201-226, S. 208.

1115 Ebd., S. 209.

1116 Maiterth, Ralf/Chirvi, Malte: Das Ehegattensplitting, S. 21-24.

1117 Vollmer, Franziska: Zur Verfassungsmäßigkeit des Ehegattensplittings, in: Sell, Barbara (Hrsg.): Ehegattensplitting und Familienpolitik, Wiesbaden 2007, S.37-53, S. 46.

1118 Tipke, Klaus: Die Steuerrechtsordnung, S. 386.

1119 Maiterth, Ralf/Chirvi, Malte: Das Ehegattensplitting, S. 23.

1120 Vollmer, Franziska: Zur Verfassungsmäßigkeit, S. 51. 
Splittingeffekt in der Realität zu 90 Prozent auf Ehepaare mit Kindern entfalle und damit typischerweise die Kindererziehung in der Ehe fördere. ${ }^{1121}$

\section{Die Beurteilung des Ehegattensplittings in der Betriebswirtschaftlichen Steuerlehre}

In der Betriebswirtschaftlichen Steuerlehre wird das Ehegattensplitting nahezu einhellig abgelehnt und als unvereinbar mit dem Grundsatz der Gleichmäßigkeit der Besteuerung angesehen. Begründet wird dies damit, dass sämtliche Entscheidungen, die außerhalb der Einkommenserzielung liegen, der privaten Lebensführung zuzuordnen seien und entsprechend keinen Einfluss auf die steuerliche Leistungsfähigkeit haben. ${ }^{1122}$

\section{Die Beurteilung des Ehegattensplittings in der Finanzwissenschaft}

In den letzten zehn Jahren hat sich die Einstellung zur Ehegattenbesteuerung deutlich gewandelt: Das Ehegattensplitting wird gegenwärtig mehrheitlich abgelehnt und die Individualbesteuerung bevorzugt. ${ }^{1123}$ Neben unerwünschten Verteilungswirkungen, fehlender Familienförderung und dem Vorwurf der Diskriminierung von Frauen resultiert diese ablehnende Haltung in erster Linie aus positiven Wirkungen auf das Arbeitsangebot der Ehefrauen sowie den damit verbundenen Wohlfahrtsgewinnen, die bei einer Individualbesteuerung erwartet werden. ${ }^{1124}$ Dabei muss jedoch differenziert werden, aus welcher Sicht Argumente für oder gegen das Ehegattensplitting vorgetragen werden.

In der „empirischen“ Finanzwissenschaft wurden in zahlreichen Studien die Arbeitsangebotseffekte der Einführung einer Individualbesteuerung sowie verschiedene Formen des Realsplittings untersucht. In einem aktuellen Beitrag wird der Frage nachgegangen, „ob das Ehegattensplitting, wie in vielen Arbeiten behauptet, einen negativen Einfluss auf das Arbeitsverhalten verheirateter Frauen hat. ${ }^{\text {"1125 }}{ }^{2}$ Die Ergebnisse dieser Arbeit „sprechen eindeutig gegen einen unmittelbaren negativen Effekt des Ehegattensplittings auf das Erwerbsverhalten von Frauen. "1126

1121 Löhr, Kerstin/Serwe, Reinhard: Das Ehegattensplitting auf dem Prüfstand. Verfassungsrecht - Unionsrecht - EMRK (Steuerwissenschaftliche Schriften), Baden-Baden 2011, S. $31 \mathrm{ff}$.

1122 Maiterth, Ralf/Chirvi, Malte: Das Ehegattensplitting, S. 26.

1123 Ebd., S. $26 f$.

1124 Ebd., S. 27. Ebd., S. 27.

1125 Chirvi, Malte: Arbeiten Frauen aufgrund des Ehegattensplittings weniger? Ein quasiexperimenteller Ansatz für Deutschland. 13. Arqus-Tagung in Berlin (Juli 2017), S. 1-39.

1126 Ebd., S. 1. 
In der normativ geprägten "traditionellen“ Finanzwissenschaft wird das Ehegattensplitting überwiegend positiv beurteilt. ${ }^{1127}$ Unter Gerechtigkeitsaspekten lässt sich die Problematik der Ehegattenbesteuerung auf die Frage verkürzen, ob das Ehegattensplitting eine Steuervergünstigung ist oder nicht. ${ }^{1128}$ Es stellt sich demgemäß die Frage nach der Höhe und der Zusammensetzung des Splittingeffekts. Die Gegner des Ehegattensplittings stellen regelmäßig auf den maximalen (absoluten) Splittingeffekt ab, der nur bei Alleinverdiener-Ehepaaren für den Bereich der sogenannten Reichensteuer mit einem Spitzensteuersatz von 45 Prozent mit einem zu versteuernden Einkommen über 501.462,- Euro zum Tragen kommt. Dieser beträgt gegenwärtig 15.761,- Euro und entspricht der Steuerersparnis eines Ehepaares gegenüber einer Individualbesteuerung. ${ }^{1129}$

Der Splittingeffekt geht deutlich zurück, sobald beide Ehegatten ihren Beitrag zum Familieneinkommen leisten, wie die folgende Abbildung zeigt:

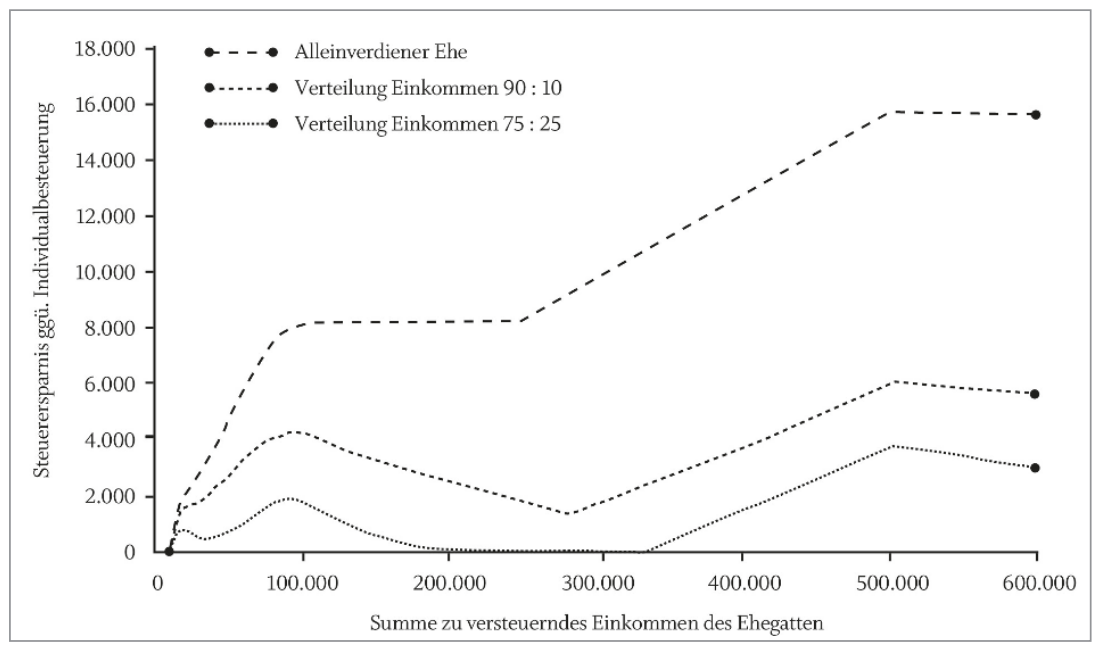

Abb. Splittingeffekt bei unterschiedlicher Verteilung des Einkommens. ${ }^{1130}$

1127 Maiterth, Ralf/Chirvi, Malte: Das Ehegattensplitting, S. 27.

1128 Scherf, Wolfgang: Das Ehegattensplitting, S. 269.

1129 Ralf Maiterth und Malte Chirvi führen aus, dass der maximale Splittingeffekt knapp zur Hälfte auf die „Reichensteuer“ zurückzuführen ist; vgl. Maiterth, Ralf/Chirvi, Malte: Das Ehegattensplitting, S. 20.

1130 Ebd., S. 20. 
Die gestrichelte Linie zeigt auf, dass bei einer Einkommensverteilung im Verhältnis 90:10 der Splittingeffekt deutlich den Maximalwert unterschreitet. Bei einem Verhältnis $75: 25^{1131}$ liegt der maximale Splittingeffekt überwiegend unter 2.000,- Euro und beträgt in der Spitze nicht mehr als 4.000,- Euro. Bei einer Gleichverteilung der Einkommen beider Ehegatten beträgt der Splittingeffekt unabhängig von der Einkommenshöhe 0 Euro. ${ }^{1132}$

Scherf verweist im Zusammenhang mit der vertikalen Gleichmäßigkeit der Besteuerung darauf, dass die Steuerentlastung durch das Splittingverfahren mit dem Einkommen absolut (aber nicht relativ) steigt, was zwangsläufig aus der Steuerprogression folgt, die in erster Linie mit dem Leistungsfähigkeitsprinzip begründet wird. Bei einem proportionalen Steuertarif gäbe es keinen sogenannten Splittingvorteil. Scherf zieht aus dieser Betrachtung das Fazit, dass derjenige, der die Einkommensteuerprogression beibehalten will, auch bereit sein sollte, die daraus resultierenden Splittingeffekte als Kehrseite der Progression hinzunehmen. ${ }^{1133}$

In der finanzwissenschaftlichen Diskussion wird von Splittingbefürwortern auf die Entscheidungen des Bundesverfassungsgerichts verwiesen, in denen auf die Ehe als Erwerbs- und Verbrauchsgemeinschaft abgestellt wird. In dem Beschluss vom 7. Mai $2013^{1134}$ bekräftigte das Bundesverfassungsgericht ausdrücklich seine früheren Argumente zugunsten des Ehegattensplittings, denn das Splitting ermögliche

den Ehegatten die freie Entscheidung, ob einer allein ein möglichst hohes Familieneinkommen erwirtschaften und sich deshalb in seinem Beruf vollständig engagieren soll, während der andere Partner den Haushalt führt, oder ob stattdessen beide Partner sowohl im Haushalt als auch im Beruf tätig sein sollen, so dass beide ihre Berufstätigkeit entsprechend beschränken. ${ }^{135}$

\subsubsection{Kritische Würdigung}

Wissenschaftlich lässt sich nicht klären, wie die „richtige“ Ehegattenbesteuerung auszusehen hat. ${ }^{1136}$

Bereits in den 1950er-Jahren wollte die Bundesregierung die Wirkung des Splittingtarifes dadurch begrenzen, dass der Unterschied in der Besteuerung des

1131 Ralf Maiterth und Malte Chirvi verweisen darauf, dass sich aus einer Einkommensteuerstatistik des Jahres 2007 in etwa diese Verteilung ergibt; vgl. Maiterth, Ralf/Chirvi, Malte: Das Ehegattensplitting, S.20, Anm. 10.

1132 Ebd., S. 20.

1133 Scherf, Wolfgang: Das Ehegattensplitting, S. 273-278.

1134 BVerfG, 2 BvR 909/06.

1135 BVerfGE 61, 319, 347.

1136 Maiterth, Ralf/Chirvi, Malte: Das Ehegattensplitting, S. 32. 
Ehepaares und des Ledigen auf einen bestimmten Höchstbetrag begrenzt wird. Die Bundesregierung hatte jedoch Bedenken, „diesen Weg zu gehen, um nicht in zahlreichen Fällen gegen die Grundsätze des Bundesverfassungsgerichts zu verstoBen“ ${ }^{1137}$ Auch der „Entwurf eines Steuerentlastungsgesetzes 1999/2000/2002“ vom November 1998 sah eine Kappung des Splittingeffektes ab dem Jahr 2002 vor. Dieser Entwurf wurde jedoch schon kurz nach seiner Veröffentlichung heftig kritisiert und nicht umgesetzt. ${ }^{1138}$ Auch in der heutigen Zeit sind die kritischen Stimmen nicht verstummt, und es wird die Frage gestellt, ob das Ehegattensplitting bei sehr hohen Einkommen "gekappt" werden kann. ${ }^{1139}$ Es stellt sich auch heute - wie in den 1950er-Jahren - die Frage, ob damit nicht gegen den Grundsatz der Besteuerung nach der Leistungsfähigkeit verstoßen würde.

Mit der an der wirtschaftlichen Leistungsfähigkeit der Ehepartner orientierten Argumentation des Beschlusses vom 7. Mai 2013 ,trägt das BVerfG zu einer Entideologisierung der Debatte bei. ${ }^{1140}$ Danach ist das Ehegattensplitting keine Steuervergünstigung, ,sondern eine mögliche gesetzgeberische Antwort auf den wirtschaftlichen Sachverhalt der ehelichen Lebensgemeinschaft. ${ }^{\text {“1141 }}$ Dem langfristig angelegten Eheversprechen scheint eine grundsätzliche Aufgaben- teilung innerhalb des Haushaltes zu folgen. ${ }^{1142}$ Die erzielten Einkünfte eines Ehepartners können entsprechend

grundsätzlich als ein Einkommen qualifiziert werden, das von beiden Ehepartnern gemeinsam erwirtschaftet worden ist, da der im Haushalt tätige Ehegatte eine ebenso verantwortungsvolle Aufgabe zu erfüllen hat und seinen berufstätigen Partner hierdurch letztlich in einem hohen Maße entlastet. ${ }^{1143}$

Das Ehegattensplitting ist auch familienfreundlich, denn es überlässt Ehepartnern mit Kindern ${ }^{1144}$ die Entscheidungsfreiheit zur Aufteilung der Erwerbs- und Erziehungszeit nach eigenen Wünschen. Je mehr Kinder im Haushalt vorhanden sind, desto mehr Zeit muss auch in die Erziehung investiert werden. Dies führt

1137 BT-Drucks. 3/260 vom 07.03.1958, S. 34.

1138 Scherf, Wolfgang: Das Ehegattensplitting, S. 271.

1139 Hey, Johanna: $\$ 3$ Steuersystem und Steuerverfassungsrecht, S. 108.

1140 Ebd., S. 109.

1141 Ebd., S. 107.

1142 Chirvi, Malte: Arbeiten Frauen aufgrund des Ehegattensplittings weniger?, S. 10.

1143 Davidsohn, Lars: Verfassungsrechtliche Würdigung, S. 109.

1144 Unterhaltsaufwendungen für Kinder werden im geltenden Einkommensteuerrecht durch den kindesbezogenen Freibetrag gemäß $\$ 32$ Abs. 6 EStG berücksichtigt. 
tendenziell auch zu einer verstärkten zeitlichen Aufteilung der Erziehung und damit $\mathrm{zu}$ wachsenden Unterschieden im Erwerbseinkommen. ${ }^{1145}$

\subsection{Zum Redistributionspostulat oder zum Grundsatz der steuerlichen Umverteilung von Einkommen und Vermögen}

Das seit der zweiten Hälfte des 19. Jahrhunderts immer stärker hervorgetretene Ideal einer steuerlichen Umverteilung von Einkommen und Vermögen ist verfassungsrechtlich grundsätzlich nicht zu beanstanden. ${ }^{1146}$ Zum einen ist in $\$ 3 \mathrm{Abs} .1$ Satz 1 Halbsatz 2 Abgabenordnung (AO) ausdrücklich enthalten, dass die Erzielung von steuerlichen Einnahmen Nebenzweck sein kann, zum anderen hat der Staat nach dem in Art. 20 Abs. 1 und Art. 28 Abs. 1 GG enthaltenen Sozialstaatsgedanken auch für eine gezielte Förderung des allgemeinen Wohlstandes zu sorgen. ${ }^{1147}$ Dabei ist jedoch zu beachten, dass eine soziale Besteuerung nach der individuellen Leistungsfähigkeit die sozialen Defizite nicht restlos auffüllen kann und daher Umverteilungsvorstellungen nur beschränkt zugänglich ist. ${ }^{1148}$

Verfassungsrechtliche Schranken einer Umverteilungspolitik sind insbesondere das Leistungsfähigkeitsprinzip und die Freiheitsrechte von Art. 12 Abs. 1 und Art. 14 Abs. 1 GG. Eine steuerrechtliche Umverteilung ist nur insoweit zulässig, als die Belastung mit Steuern noch als verhältnismäßig und gleichmäßig bezeichnet werden kann. ${ }^{1149}$

1145 Deutsches Steuerzahlerinstitut des Bundes der Steuerzahler e. V.: Bausteine für eine Reform des Steuersystems. Das DSI-Handbuch Steuern, Berlin 2013. S.99f.

1146 Tipke, Klaus: Die Steuerrechtsordnung, S. 404-416, S. 416.

1147 Paulick, Heinz: Die wirtschaftspolitische Lenkungsfunktion des Steuerrechts und ihre verfassungsmäßigen Grenzen, in: Haller, Heinz u. a. (Hrsg.): Theorie und Praxis des finanzpolitischen Interventionismus. Fritz Neumark zum 70. Geburtstag, Tübingen 1970, S. 203-228, S. 217.

1148 Elschen stellt die Frage, ob Umverteilungsmaßnahmen nicht besser im Sozial- und Transferrecht aufgehoben sind als im Steuerrecht; denn „das ganze ,System ' der Verknüpfung von Sozialleistungen und Sozialausgaben mit der Besteuerung und diversen steuerlichen Einkommensbegriffen ist völlig beliebig, ohne jeden systemtragenden Grundsatz." Elschen, Rainer: Entscheidungsneutralität, Allokationseffizienz und Besteuerung nach der Leistungsfähigkeit. Gibt es ein gemeinsames Fundament der Steuerwissenschaften?, in: Steuer und Wirtschaft 68 (1991), S. 99-115, S. 113 f.

1149 Davidsohn, Lars: Verfassungsrechtliche Würdigung, S. 203-228, S. 227. 
Zur Herbeiführung einer steuerrechtlichen Umverteilungspolitik kommen die folgenden Instrumente in Betracht: ${ }^{1150}$ ein progressiver Einkommensteuertarif, eine Besteuerung des Vermögens und die Erbschaftsteuerbelastung.

\subsubsection{Umverteilende Steuerpolitik mithilfe eines progressiven Einkommensteuertarifs}

Der Gesetzgeber hat bei der Festlegung des Einkommensteuertarifs einen weiten Ermessensspielraum, da das Grundgesetz keinen konkreten Verlauf des Tarifs vorschreibt. ${ }^{1151}$ Der progressive Tarif soll sozialstaatliche Umverteilungsgerechtigkeit verwirklichen. Diese beabsichtigte Wirkung ,ist ein bewusstes Negieren des Neutralitätsprinzips der Besteuerung im Sinne der Forderung ,leave them as you find them."'1152 $\mathrm{Zu}$ beachten hat der Gesetzgeber jedoch insbesondere die Begrenzungen, die durch das Grundgesetz in Art. 3 Abs. 1, das Leistungsfähigkeitsprinzip, das Sozialstaatsprinzip, die Garantie des Eigentums und durch die Freiheitsgrundrechte nach Art. 12 Abs. 1 und Art. 2 Abs. 1 GG festgelegt worden sind. Um hohe progressive Einkommensteuertarife zu rechtfertigen, muss ein erhebliches Bedürfnis nach einer Einkommensumverteilung vorliegen, z. B. wenn die Verteilung der einzelnen Einkünfte auffällige und extreme Ungleichheiten aufweist. Der Gesetzgeber muss jedoch darauf achten, dass dem Steuerpflichtigen jederzeit ein angemessener Spielraum verbleibt, um die von ihm beabsichtigten und für seine berufliche Zukunft notwendigen Vorhaben durchführen zu können. ${ }^{1153}$

1150 Neumark, Fritz: Grundsätze der Besteuerung in Vergangenheit und Gegenwart, 2. Aufl., Wiesbaden 1970, S. 50.

1151 Das Leistungsfähigkeitsprinzip fordert keinen progressiven Tarif, vgl. auch BVerfGE 115, 97 (117).

1152 Bareis, Peter: Zur Problematik steuerjuristischer Vorgaben für die Einkommensteuer - Tarifstruktur und Familienplitting als Musterbeispiele, in: Deutsches Steuerrecht 11 (2010), S. 565-573, S. 567; Hey, Johanna: $\$ 8$ Einkommensteuer, in: Tipke, Klaus/Lang, Joachim (Hrsg.): Steuerrecht, fortgeführt von Roman Seer u.a., 22. Aufl., Köln 2015, S. 283-484, S. 461.

1153 Davidsohn, Lars: Verfassungsrechtliche Würdigung, S. 231-235. 


\subsubsection{Umverteilende Steuerpolitik durch die Besteuerung des Vermögens}

Als ein wirksames steuerpolitisches Instrument, um eine volkswirtschaftlich nützliche Umschichtung der Vermögen zu bewirken, betrachtete Fritz Karl Mann die regelmäßig zu erhebende Vermögensteuer (VSt). ${ }^{1154}$ Dieser Meinung stand jedoch die Finanzwissenschaft des 18. und 19. Jahrhunderts ablehnend gegenüber, ${ }^{1155}$ bis im letzten Drittel des 19. Jahrhunderts die Vermögensteuer wieder eine breitere Akzeptanz fand. ${ }^{1156}$

Das preußische Einkommensteuergesetz von 1891 wurde im Jahr 1893 durch eine Vermögensteuer ergänzt, die in den darauffolgenden Jahren von einem Großteil der deutschen Bundesstaaten übernommen wurde.

Die verfassungsrechtlichen Grenzen einer gezielten umverteilenden Vermögensteuer ergeben sich vor allem aus Art. 14 Abs. 1 GG. Eine fixe Grenze ist aus dem Grundgesetz jedoch nicht zu entnehmen. Vielmehr muss grundsätzlich durch einen Abwägungsprozess ermittelt werden, ob sowohl das Grundrecht auf Eigentum als auch das Sozialstaatsprinzip hinreichende Berücksichtigung finden. ${ }^{1157}$

Das Bundesverfassungsgericht hat mit Beschluss vom 22. Juni $1995 \$ 10 \mathrm{Nr} .1$ des Vermögenssteuergesetzes (VStG) in der Fassung vom 14. September 1994 in allen seinen seitherigen Fassungen mit Art. 3 Abs. 1 GG als unvereinbar erklärt. ${ }^{1158}$ Aufgrund dieses Beschlusses kann die Vermögensteuer wegen ihrer teilweisen Verfassungswidrigkeit seit 1997 nicht mehr erhoben werden. ${ }^{1159}$

Im Gegensatz zur Vermögensteuer, deren Aufkommen den Ländern zusteht (Art. 106 Abs. 1 Nr. 1 GG), stehen die einmaligen Vermögensabgaben dem Bund zu (Art. 106 Abs. 1 Nr. 5 GG). Die einmaligen Vermögensabgaben können als Ultima Ratio dann in Betracht kommen, wenn zur Finanzierung des unbedingt notwendigen Staatsbedarfs keine anderweitigen, weniger einschneidenden steuerrechtlichen

1154 Mann, Fritz Karl: Vermögensteuer, in: Elster, Ludwig/Weber, Adolf/Wieser, Friedrich (Hrsg.): Handbuch der Staatswissenschaften 8, 4. Aufl., Jena 1928, S. 608-620, S. 613.

1155 Fux, Boleslav: Die Vermögensteuer, in: Gerloff, Wilhelm/Meisel, Franz (Hrsg.): Handbuch der Finanzwissenschaft, Bd. 2, Besondere Steuerlehre, Finanzwissenschaft der öffentlichen Körperschaften, Der öffentliche Kredit, Tübingen 1927, S. 133-158, S. 135.

1156 Strutz, Georg: Die Vermögensteuer, in: Ders. (Hrsg.): Handbuch des Reichssteuerrechts. Systematische Darstellung der Steuergesetze (einschließlich Zollrecht) des Deutschen Reiches, 3. Aufl., Berlin/Wien 1927, S.619-673, S. 623.

1157 Davidsohn, Lars: Verfassungsrechtliche Würdigung, S. 253.

1158 BVerfG, 22.06.1995 - 2 BvL 37/91.

1159 VStG: in: Steuergesetze, Textsammlung mit Verweisungen und Sachverzeichnis, Loseblattsammlung, S. 1 und Anmerkung zu $\$ 10$ VStG. 
Möglichkeiten zur Verfügung stehen. Das im Jahr 1919 eingeführte „Reichsnotopfer" mit einem Höchststeuersatz von 65 Prozent wäre durch die durch den Krieg verursachten Verteilungsungerechtigkeiten nach heutigem Verfassungsrecht als eine zulässige finanzpolitische Nachkriegsmaßnahme zu qualifizieren gewesen. ${ }^{1160}$

\subsubsection{Umverteilende Steuerpolitik durch Erbschaftsteuerbelastung}

Das Grundgesetz schließt eine umverteilend wirkende Erbschaftsteuer nicht generell aus, doch darf das Erbrecht nach Art. 14 Abs. 1 GG und Art. 19 Abs. 2 GG in seinen Grundlagen nicht berührt werden. ${ }^{1161}$

Der Erwerb einer Erbschaft erhöht die wirtschaftliche und steuerliche Leistungsfähigkeit des Erben, weil dieser durch die Erbschaft Einkommen für die private Lebensführung erlangt. Die Belastung der Erbschaftsteuer auf das beim Erblasser versteuerte Vermögen bedeutet keine Doppelbelastung desselben Steuerpflichtigen, weil Erblasser und Erbe nicht eine Person sind. Mit der Erbschaftsteuer soll allein die beim Erwerber durch den Erbanfall neu entstandene steuerliche Leistungsfähigkeit erfasst werden. ${ }^{1162}$ Neben dem im Leistungsfähigkeitsprinzip konkretisierten Fiskalzweck kann eine gesonderte Erb- und Schenkungsteuer zusätzlich den sozialoder wirtschaftspolitischen Zweck einer Umverteilung erfüllen. ${ }^{1163}$

Im Hinblick auf das Grundrecht des Art. 6 Abs. 1 GG sind einer umverteilenden Erbschaftsteuer deutliche Grenzen gesetzt. Danach stehen Ehe und Familie unter dem besonderen Schutz des Staates, der auf die Kontinuität der Ehe- und Familiengüter achten muss und ihre Erhaltung nicht gefährden darf. ${ }^{1164}$ Der Familienbesitz ist bis zu einem gewissen Grad durch den verfassungsrechtlichen Schutz von Ehe und Familie von der Erbschaftsteuer freizustellen, sodass die Erbschaftsteuer als Instrument einer gezielten Vermögensumverteilung in diesem Fall nicht zum Zuge kommen kann. ${ }^{1165}$

1160 Davidsohn, Lars: Verfassungsrechtliche Würdigung, S. 261-263.

1161 Ebd., S. $279 \mathrm{f}$.

1162 Jansen, Harald: Erbschaftsteuerliche Wirkungen bei Vermögensübertragungen. Entscheidungswirkungen und Gleichmäßigkeit der Besteuerung, Berlin 2006, S. $43 \mathrm{f}$.

1163 Ebd., S. 45 f.; Hey, Johanna: $\$ 3$ Steuersystem und Steuerverfassungsrecht, S. 119; Dies.:

$\$ 7$ Einführung in das besondere Steuerschuldrecht, S. 259.

1164 Vgl. hierzu BVerfGE 93, S. 142.

1165 Davidsohn, Lars: Verfassungsrechtliche Würdigung, S. $275 \mathrm{ff}$. 
Der Gesetzgeber muss auch sicherstellen, dass die Existenz von kleineren und mittelständischen Betrieben durch die Erbschaftsteuer nicht gefährdet wird. ${ }^{1166}$ Eine Erbschaftsteuerlast darf eine vom Erben beabsichtigte Fortführung eines Betriebes nicht gefährden. ${ }^{1167}$ Eine weitgehende steuerliche Freistellung von betrieblich gebundenem Erbvermögen stellt keinen Verstoß gegen Art. 3 Abs. 1 GG dar; denn Betriebe sind in einem hohen Maße gemeinwohlgebunden und gemeinwohlverpflichtet. Sie sind nicht nur Garant für die Erhaltung der Arbeitsplätze, sondern auch unerlässlich für die gesamtwirtschaftliche Produktivität und Wertschöpfung. ${ }^{1168}$

Allerdings führt die Befreiung Einzelner in der Regel zu einer Mehrbelastung anderer und bedeutet, dass die Privilegierten Liquiditätsvorteile erhalten, die nachteilige Wettbewerbsverzerrungen zur Folge haben können. Unabhängig davon ist auch fraglich, ob die Ausübung unternehmerischer Funktionen in einer marktwirtschaftlichen Ordnung eine steuerlich schonende Übertragung von Vermögen auf die nächste Generation erfordert. ${ }^{1169}$

\subsection{Die Steuerpolitik in der Bundesrepublik Deutschland}

Seit dem Bestehen der Bundesrepublik Deutschland ab 1949 war die Finanzpolitik in viel höherem Maße als in früheren Perioden wirtschaftspolitisch orientiert. Vorrangiges Ziel der Regierung waren die Wiederankurbelung der deutschen Wirtschaft und die Förderung des wirtschaftlichen Wachstums. ${ }^{1170}$ Der Wirtschaftswissenschaftler Hans Möller, der 1948 bei der Einführung der DM und damit indirekt auch beim Lastenausgleich mitwirkte, begründete die Bevorzugung der Steuer als Lenkungsinstrument gegenüber der Gerechtigkeit rückblickend wie folgt: „Mit diesem Verzicht auf größere Verteilungsgerechtigkeit war jedoch ein Effizienzgewinn in Form höherer volkswirtschaftlicher Wachstumsraten verbunden. " ${ }^{1171}$

1166 Vgl. hierzu BVerfGE 93, S. $175 \mathrm{f}$.

1167 Ebd., S. 176.

1168 Ebd., S. 175 f.; Davidsohn, Lars: Verfassungsrechtliche Würdigung, S. 282 f.

1169 Zur kritischen Analyse von erbschaftsteuerlichen Vergünstigungen zur Schonung von Unternehmungen und von Familien vgl. Hansen, Harald: Erbschaftsteuerliche Wirkungen bei Vermögensübertragungen, S. 163-228.

1170 Ehrlicher, Werner: Finanzpolitik seit 1945, S. 216; Schremmer, Eckart: Über „gerechte Steuern“, S. 38; Davidsohn, Lars: Verfassungsrechtliche Würdigung, S. 325.

1171 Möller, Hans: Die Währungsreform von 1948 und die Wiederherstellung marktwirtschaftlicher Verhältnisse, in: Hampe, Peter (Hrsg.): Währungsreform und Soziale Marktwirtschaft. Rückblicke und Ausblicke, München 1989, S. 55-77, S. 69 ff. 
Ludwig Erhard hat seine Zielsetzung der Wohlstandsmehrung durch Expansion damit begründet, dass es „ungleich sinnvoller [sei], alle einer Volkswirtschaft zur Verfügung stehenden Energien auf die Mehrung des Ertrages der Volkswirtschaft zu richten als sich in Kämpfen um die Distribution des Ertrages zu zermürben. "1172 Die verteilungspolitische Unausgewogenheit der in den Jahren nach 1949 vorgenommenen steuerpolitischen Maßnahmen standen jedoch vielfach in Kritik, da diese zu einer einseitigen Vermögensbildung in der Bevölkerung führten und in der Wirtschaft zunehmend Konzentrationstendenzen verursachten. ${ }^{1173}$

Der neue Staatszweck, dem das Steuerrecht angepasst wurde, war jetzt Lenkung und Steuerung, weniger die Umverteilung. Die Funktion der Steuer hatte sich verändert: Die Frage war nicht mehr - oder allenfalls nachrangig - ob die Steuern "gerecht" waren, sondern ob diese wirtschaftspolitisch "richtig" oder "nicht richtiger", „hilfreicher" oder nicht „hilfreicher" waren. ${ }^{1174}$ Damit verlor die Einkommensteuer als Instrument der sozialen Umverteilung erheblich an Gewicht verglichen mit der Aufgabe, die ihr Finanzminister Matthias Erzberger 1920 zugewiesen hatte. ${ }^{1175}$

Für die Darstellung der Entwicklung der Steuerpolitik bieten sich die Zäsuren der Wirtschaftsentwicklung an, wie sie in den Rezessionen 1966/67 und 1981/82 vorgegeben sind. ${ }^{1176}$

\subsubsection{Die Steuerpolitik in der Wiederaufbauphase ab 1948}

In der Wiederaufbauphase lassen sich drei Abschnitte unterscheiden, bei denen sich die Fördermaßnahmen deutlich in ihrer Orientierung unterscheiden: ${ }^{1177}$

Zunächst stand die Förderung der Selbstfinanzierung im Vordergrund. $\mathrm{Zu}$ erwähnen ist das Militärgesetz Nr. 64 vom 20. Juni 1948, das die Absetzung der Hälfte des nicht entnommenen Gewinns bis zu einer gewissen Höchstgrenze der Bemessungsgrundlage erlaubte. Bedeutsam waren auch die zusätzlichen Abschreibungsmöglichkeiten für die verschiedensten Anlagen und Branchen. Mit dem Investitionshilfegesetz vom 7. Januar $1952^{1178}$ wurde dem Kohle- und Eisenerzbau erlaubt, für die Jahre 1952 bis 1954 zusätzliche Sonderabschreibungen bis zu 50

1172 Erhard, Ludwig: Wohlstand für Alle (1957), 8. Aufl., Düsseldorf 1964, S. 10.

1173 Davidsohn, Lars: Verfassungsrechtliche Würdigung, S. $337 \mathrm{f}$.

1174 Schremmer, Eckart: Über „gerechte Steuern“, S. 27.

1175 Ebd., S. 38.

1176 Ehrlicher, Werner: Finanzpolitik seit 1945, S. 213 ff.

1177 Ebd., S. 219.

1178 Gesetz über die Investitionshilfe der gewerblichen Wirtschaft, BGBl., I 1952, S 7 ff. 
Prozent der Anschaffungs- oder Herstellungskosten vorzunehmen mit der Maßgabe, dass die hierdurch frei gewordenen finanziellen Mittel wieder reinvestiert würden und einer Steigerung der Produktion dienten. ${ }^{1179}$

Besonders weitgehende Vergünstigungen schuf das DM-Eröffnungsbilanzgesetz vom 21. August 1949, das den Unternehmen erlaubte, bereits vollständig abgeschriebene Wirtschaftsgüter wieder bis zu einem Drittel ihres Neuwertes in der Bilanz anzusetzen. ${ }^{1180}$

Mit dem Einkommensteueränderungsgesetz vom 27. Juni $1951^{1181}$ wurde eine Reihe von Begünstigungen der Selbstfinanzierung abgeschafft oder eingeschränkt, da die Förderung der Selbstfinanzierung zu erheblichen Gewinnen vor allem in den Investitionsgüterindustrien geführt hatte. ${ }^{1182}$ Dagegen wurde die Fremdfinanzierung durch das Gesetz zur Förderung des Kapitalmarkts vom 15. Dezember $1952^{1183}$ über eine niedrigere Besteuerung der Zinserträge gefördert. Einerseits wurde bei Einkünften aus Kapitalvermögen die progressive Einkommensteuer durch eine Kapitalertragsteuer von 30 Prozent ersetzt, andererseits wurden bestimmte Anlageformen vollständig von der Steuer befreit. ${ }^{1184}$ Die steuerlichen Vergünstigungen für das Kontensparen und den Erwerb festverzinslicher Wertpapiere trugen zu einer spürbaren Belebung des Kapitalmarktes bei. ${ }^{1185} \mathrm{Zu}$ bedenken ist jedoch, dass in erster Linie die oberen Einkommensklassen von der Einführung der ermäßigten linearen Zinsbesteuerung in Höhe von 30 Prozent profitierten. ${ }^{1186}$

Im Interesse einer allgemeinen Förderung der Sparfähigkeit wurden zunächst im Rahmen der sogenannten kleinen (1953) und sodann der großen (1954) Steuerreform die Steuersätze erheblich gesenkt. Mit der „Großen Steuerreform“vom 16.

1179 Muscheid, Jutta: Die Steuerpolitik in der Bundesrepublik Deutschland 1949-1982. Volkswirtschaftliche Schriften 365 (1986), S. 51.

1180 Vgl. dazu Merkle, Franz: „DM-Eröffnungsbilanz“, in: Köhler, Richard u.a. (Hrsg.): Handwörterbuch der Betriebswirtschaft, Bd. 1, 3. Aufl., Stuttgart 1956, Sp. 1448; Pehl, Günter: Steuerpolitik in der Bundesrepublik Deutschland, Köln 1962, S. 65.

1181 BGBl. I 1951, S. 411.

1182 Ehrlicher, Werner: Finanzpolitik seit 1945, S. 220.

1183 BGBl. I 1952, S. 793.

1184 Stücken, Rudolf: Deutsche Geld- und Kreditpolitik 1914 bis 1963, 3. Aufl., Tübingen 1964, S. 237 f.

1185 Ebd., S. 239.

1186 Davidsohn, Lars: Verfassungsrechtliche Würdigung, S. 329. 
Dezember $1954^{1187}$ wurde - neben der Anhebung einer Reihe von Freibeträgen - der Einkommensteuerspitzensatz von 70 Prozent auf 55 Prozent gesenkt. ${ }^{1188}$

Trotz dieser Steuerermäßigungen und -erleichterungen setzte sich in den steuerpolitischen Diskussionen Anfang der Fünfzigerjahre die Erkenntnis durch, dass die einzelnen Steuern keineswegs harmonisch zusammenwirkten, sondern ein unsystematisches Durch-, Neben- und Gegeneinander darstellten. Der Ruf nach einer großen „Organischen Steuerreform“ war infolgedessen nicht mehr zu überhören. ${ }^{1189}$ Dies schlug sich in zahlreichen Gutachten, Stellungnahmen und Untersuchungen nieder, die zwar im Einzelnen voneinander abwichen, allerdings war bei den grundsätzlichen Schwerpunkten eine gewisse Einmütigkeit zu beobachten. Haushaltspolitische Bedenken und interessensbedingte Widerstände verhinderten jedoch die organische Steuerreform, die in der Zeit der Haushaltsüberschüsse eine einzigartige Chance für einen Neuanfang hätte sein können. ${ }^{1190}$

In den Jahren 1957 bis 1961 wurde die Kapitalbildung gezielt gefördert, insbesondere das Sparen von Arbeitnehmern unterstützt, und Maßnahmen zur Förderung des Mittelstandes, für Zonenrandgebiete, für Berlin, für den Kohlenbergbau und den Agrarsektor wurden ergriffen. Der expandierende Wohlfahrtsstaat schuf - Mitte der Sechzigerjahre - mit höheren Sozialleistungen, Mehrausgaben für Landesverteidigung, Bundesbahn und Bergbau sowie für Wirtschaftsförderung und Infrastruktur neue Ausgaben. Dazu kam, dass in der Renten- und Unfallversicherung der Versichertenkreis vergrößert, das Wohn- und Kindergeld ausgebaut und die Sozialhilfe umgestaltet wurde. In der Besteuerungspolitik rückten verteilungspolitische Motive in den Vordergrund, das "Splittingverfahren“ wurde neu geregelt und die „Vermögensbildung in Arbeitnehmerhand“ gefördert. ${ }^{1191}$

\subsubsection{Das Keynesianische Konzept der Globalsteuerung ab 1967}

1966 glitt die Wirtschaft in die Rezession ab; die Bundesregierung lehnte es ab, lenkend in den Wirtschaftsprozess einzugreifen. Erst die Große Koalition verhalf der „Fiscal policy“ zum Durchbruch: Der neue Staatszweck, dem das Steuersystem

1187 Gesetz zur Neuordnung von Steuern vom 16.12.1954, BGBl. I 1954, S. 373.

1188 Ehrlicher, Werner: Finanzpolitik, S. 220 f.

1189 Schmölders Günter: Geschichte der Besteuerung, S. $624 \mathrm{f}$.

1190 Ebd., S. 625.

1191 Ehrlicher, Werner: Finanzpolitik, S. $221 \mathrm{f}$. 
angepasst werden sollte, war jetzt Lenkung, Steuerung und weniger die Umverteilung. ${ }^{1192}$

Seit dem Godesberger Programm von 1959 waren sowohl die Sozialdemokraten als auch die Gewerkschaften für eine global gesteuerte Marktwirtschaft eingetreten, die in der Folge durch eine starke Steigerung der Staatsquote gekennzeichnet war. Die sozial-liberale Koalition war von dem Glauben getragen gewesen, dass der Staat alles viel besser kenne und könne. In der Regierungserklärung von Bundeskanzler Brandt vom 28. Oktober 1969 wurden großzügige Vorhaben für alle Ressorts angekündigt und in der Folge trugen Bund, Länder und Gemeinden gleichermaßen zur Expansion der öffentlichen Ausgaben in einem bis dahin nicht gekannten Ausmaß bei.

Seit 1973 wuchs der Schuldenberg der öffentlichen Haushalte von 126 Milliarden DM im Jahr 1970, über 256 Milliarden DM im Jahr 1975, auf 615 Milliarden DM im Jahr 1982, verfünffachte sich also fast in diesem Zeitraum. ${ }^{1193}$ Die anhaltende Diskrepanz zwischen Ausgaben und Steuereinnahmen führte zu einer erheblichen Zunahme des Schuldenstandes der öffentlichen Gebietskörperschaften.

In der Regierungserklärung vom 28. Oktober 1969 wurde auch das Vorhaben verkündet, ein gerechtes, einfaches und überschaubares Steuersystem zu schaffen. ${ }^{1194}$ Bundesfinanzminister Karl Schiller erläuterte das Ziel folgendermaßen:

Das Steuerrecht, die Besteuerung der Bürger, soll einfacher und übersichtlicher, soll vor allem gerechter werden (...) Diese Reform soll nach dem Willen der Bundesregierung so viel soziale Gerechtigkeit wie irgend möglich verwirklichen. ${ }^{1195}$

An der Diskrepanz zwischen den Vorstellungen der SPD zur Ausgabenseite und den Vorstellungen der FDP zur Einnahmenseite ist die sozialliberale Koalition letztlich politisch gescheitert. Führende Finanzpolitiker, die ihre sachgerechten Vorstellungen durchsetzen wollten, wurden zur Aufgabe veranlasst. Finanzminister Möller legte sein Amt im Jahr 1971 nieder, und Wirtschafts- und Finanzminister Schiller trat

1192 Schremmer, Eckart: Über „gerechte Steuern“, S. 27.

1193 Ullmann, Hans-Peter: Der deutsche Steuerstaat, S. $202 \mathrm{f}$.

1194 Brandt, Willy: Regierungserklärung von Bundeskanzler Willy Brandt vor dem Deutschen Bundestag in Bonn am 28. Oktober 1969 auf: http://www.willy-brandt.de/fileadmin/ brandt/Downloads/Regierungserklaerung_Willy_Brandt_1969.pdf (letzter Zugriff: 17.02.2017). Verhandlungen des Deutschen Bundestages, Stenographische Berichte, Bd. 71, S. 24 B/C und BT-Drucks. VI/1152, S. 2 unter II und 7/78.

1195 o. V.: Bulletin der Bundesregierung vom 23.6.1971. 
im Jahr 1972 zurück. Letztendlich scheiterte auch Bundeskanzler Helmut Schmidt an den ungelösten Problemen der Finanz- und Steuerpolitik dieser Periode. ${ }^{1196}$

\subsection{3 „Mehr Markt, weniger Staat“. Neoklassisch-liberale Steuerpolitik ab 1982}

„Mehr Markt, weniger Staat" und „über geordnete Finanzen zu einem geordneten Staat", so lauteten 1982 die Zielsetzungen der neu gewählten christdemokratisch-liberalen Koalitionsregierung unter Bundeskanzler Helmut Kohl. Die Steuerpolitik sollte die Hemmungen des wirtschaftlichen Wachstums beseitigen und die wirtschaftliche Aktivität möglichst fördern nach dem Motto: „Leistung soll sich wieder lohnen.“

Die Entwicklung in den Achtzigerjahren wurde dadurch begünstigt, dass es sich in der Zeit bis 1991 um den längsten anhaltenden Wirtschaftsaufschwung in der Nachkriegszeit handelte. Die realen Wachstumsraten betrugen 1,5 bis 3,1 Prozent, sodass die Steuereinnahmen überproportional stiegen und die Ausgaben unterproportional zurückblieben. Die Koalition setzte ab Mitte der Achtzigerjahre auf steigende Einnahmen und reformierte die Einkommensteuer. Diese sollte vor allem den „Mittelstandsbauch“ abbauen, Mehrleistung belohnen und Steuerflucht und Schattenwirtschaft eindämmen.

Mit dem Steuerpaket von 1990 wurde der Grundfreibetrag erhöht, der Eingangssatz von 22 Prozent auf 18 Prozent, der Spitzensteuersatz von 56 Prozent auf 53 Prozent und der Körperschaftsteuersatz von 56 Prozent auf 50 Prozent gesenkt. Gleichzeitig wurde eine große Zahl von Steuersubventionen und Sonderregelungen abgebaut. ${ }^{1197}$

Um die Steuerreform 1990 wurde auf allen Etappen ihrer Verabschiedung heftig gekämpft. Wieder einmal hieß es: „Ziel ist ein gerechtes und einfacheres Steuersystem“. ${ }^{1198}$ Dabei ging es immer um die Priorität bzw. den Ausgleich zwischen wachstumspolitischer Orientierung einerseits und verteilungs- und familienpolitischer Orientierung andererseits. ${ }^{1199}$

Besonders heftig war die Auseinandersetzung über die Senkung des Spitzensteuersatzes der Einkommensteuer. Der Sachverständigenrat führte hierzu das Folgende aus:

1196 Ehrlicher, Werner: Finanzpolitik, S. 233.

1197 Ebd., S. 233-239.

1198 BT-Drucks. 11/2226, S. 1 unter A: Zielsetzung.

1199 Ehrlicher, Werner: Finanzpolitik, S. 238. 
Gerade die Kontroverse über die Senkung des Spitzensatzes hat demonstriert, wie sehr in der Bundesrepublik wachstumsfördernde Reformen durch verteilungspolitische Rücksichtnahme erschwert werden [...]. Die Besteuerung der Spitzeneinkommen hat in verteilungspolitischer Hinsicht eher optische Bedeutung, denn deren Aufkommen erbringt nur relativ wenig Umverteilungsmasse. Umso bedeutsamer sind die Spitzensteuersätze für die Wachstumspolitik, da sie vielfach die für unternehmerische Entscheidungen relevanten Grenzsteuersätze sind. ${ }^{1200}$

Die vielen Kompromisse im Hinblick auf verteilungs- und familienpolitische Forderungen verhinderten eine klare und eindeutige Ausrichtung der Steuerpolitik auf Förderung des Wachstums und der Beschäftigung, wie sie mit großem Erfolg - doch auch mit weniger erwünschten Nebenwirkungen - in der ersten Nachkriegsperiode betrieben worden war.

Die gesamtwirtschaftliche Entwicklung in den Neunzigerjahren wurde von den Folgen der Wiedervereinigung geprägt. Die Lasten im Zusammenhang mit der Wiedervereinigung wurden mit Krediten finanziert, was zu einem erheblichen Anstieg des Schuldenstandes des Staates führte. Da die „Maastricht-Kriterien“ erfüllt werden mussten, gerieten die öffentlichen Haushalte unter Konsolidierungsdruck, der erst Ende der Neunzigerjahre nachließ. Der wirtschaftliche Aufschwung ließ die Einnahmequellen ansteigen, sodass die Neuverschuldung verringert werden konnte und umfangreiche Steuerentlastungen in mehreren Etappen durchgeführt werden konnten. ${ }^{1201}$

Die Jahre nach 2000 waren von einer hartnäckigen wirtschaftlichen Stagnation mit hoher Arbeitslosigkeit geprägt. Die Folge waren geringere Steueraufkommen gepaart mit höheren Sozialausgaben. Erst im Jahresverlauf 2000 „drehte“ die Konjunktur, die positive Entwicklung der Exportindustrie sowie ein aufgestauter Modernisierungsbedarf gaben der privaten Investitionstätigkeit Impulse. ${ }^{1202}$

1200 Sachverständigenrat, Jahresgutachten 1989/1990 des Sachverständigenrates zur Begutachtung der gesamtwirtschaftlichen Entwicklung, BT-Drucks. 11/5786 vom 23.11.1989, S. $115 f$.

1201 Vesper, Dieter: Finanzpolitische Entwicklungstendenzen und Perspektiven des Öffentlichen Dienstes in Deutschland, Gutachten im Auftrag des Instituts für Makroökonomie und Konjunkturforschung in der Hans-Böckler-Stiftung, Berlin 2012, S $4 \mathrm{f}$.

1202 Ebd., S. 6. 


\subsubsection{Wiedergeburt der antizyklischen Finanz- und Steuerpolitik nach 2009}

Mit dem Ausbruch der Finanzkrise in den Vereinigten Staaten von Amerika im Jahr 2007, der eine globale Wirtschaftskrise im Herbst 2008 folgte, wurde der gesamte europäische Raum in Mitleidenschaft gezogen. Die Insolvenz der großen amerikanischen Investmentbank Lehman Brothers und die Sorge vor weiteren Zusammenbrüchen ergriff weite Teile des globalen Finanzsystems; Banken scheuten sich, überhaupt Kredite zu vergeben. Die allgemeine Unsicherheit erfasste die gesamte Wirtschaft; Unternehmen stellten ihre Investitionen zurück oder konnten mangels Bankkrediten die geplanten Investitionen nicht finanzieren. Dies löste rund um den Globus einen starken Einbruch der Wirtschaftstätigkeit aus. Um die Finanz- und Wirtschaftskrise einzudämmen, beschlossen die zwanzig wichtigsten Wirtschaftsnationen der Welt sowie weitere globale Institutionen zahlreiche Programme. ${ }^{1203}$

Der Sachverständigenrat zur Begutachtung der gesamtwirtschaftlichen Entwicklung führte in seinem Jahresgutachten 2009/10 aus, dass das deutsche Konjunkturprogramm im europäischen Vergleich eine herausgehobene Stellung eingenommen habe. Die Konjunkturprogramme hätten „die gesamtwirtschaftliche Nachfrage stabilisiert und einen noch stärkeren Einbruch des Bruttoinlandsprodukts verhindert. Ohne sie wäre alles noch schlimmer gekommen."

\subsection{Zusammenfassung und Fazit}

In der Wiederaufbauphase nach dem Zweiten Weltkrieg wurden verschiedene Fördermaßnahmen ausgeführt, die sich deutlich in ihrer Orientierung unterschieden. Aufgrund der auf Selbstfinanzierung ausgerichteten Steuerpolitik des Bundesministers der Finanzen, Fritz Schäffer, nahm das Wirtschaftswachstum derart zu, dass sich die Steuereinnahmen von 1950 bis 1965 mehr als verdreifachten. Darüber hinaus betrieb Fritz Schäffer eine antizyklische Finanzpolitik, da

1203 Bundesbank: Die Finanz-, wirtschafts- und Staatsschuldenkrise. Ein kurzer Überblick, o. D. https://www.bundesbank.de/Redaktion/DE/Dossier/Service/schule_und_bildung_kapitel_4.html?notFirst=true\&docId=147560\#doc147560bodyText1 (letzter Zugriff: 02.03.2017).

1204 Sachverständigenrat zur Begutachtung der gesamtwirtschaftlichen Entwicklung: „Die Zukunft nicht aufs Spiel setzen“, Jahresgutachten 2009/10, veröffentlicht am 13.11.2009, auf: https://www.sachverstaendigenrat-wirtschaft.de/jahresgutachten-2009-2010.html (letzter Zugriff: 17.02.2017), S. 165. 
die nicht sofort benötigten Steuereinnahmen bei der Bundesbank angesammelt, jedoch später durch großzügige Wahlgeschenke in Form von niedrigeren Steuern, höheren Subventionen und Sozialleistungen wieder ausgegeben wurden. In den Jahren zwischen 1957 und 1961 wurde die Kapitalbildung gezielt gefördert und verteilungspolitische Motive rückten in den Vordergrund.

Die Wirtschaft glitt 1966 in die Rezession ab. Hervorgerufen wurde dies sowohl durch die Vermeidung antizyklischer, konjunkturpolitischer Maßnahmen als auch durch den fortschreitenden restriktiveren Kurs der Bundesbank. Erst die Große Koalition verhalf der „Fiscal policy“ 1967 zum Durchbruch und bekämpfte die Rezession mit Investitionsprogrammen.

In der Steuerpolitik ab 1969 ging es darum, so viel soziale Gerechtigkeit wie irgend möglich zu verwirklichen; die Grund-, Vermögen-, Erbschaft-, Gewerbeund insbesondere die Einkommensteuer wurden in der Steuerreform von 1974/75 umgestaltet. Erst in der zweiten Hälfte der Siebzigerjahre wurden die wirtschaftspolitischen den verteilungspolitischen Zielen vorgezogen, eine klare steuerpolitische Linie war jedoch nicht zu erkennen.

„Leistung soll sich wieder lohnen“, so lautete die Zielsetzung der christdemokratisch-liberalen Koalitionsregierung ab 1982. Mehrleistung sollte belohnt, der sogenannte Mittelstandsbauch, die Steuerflucht und die Schattenwirtschaft abgebaut werden.

Die schwarz-gelbe Koalition von 2009 wollte die Leistungsbereitschaft der Bürgerinnen und Bürger stärken und für Unternehmen Rahmenbedingungen schaffen, die diesen in Zeiten der Globalisierung eine starke Stellung ermöglichen sollten. Die vielen Vorschläge aus den Koalitionsfraktionen sind jedoch nicht umgesetzt worden. Die Reform der Gemeindefinanzen, als „Mega Reform“ angekündigt, scheiterte nach einjährigen Beratungen einer Kommission mit den Ländern und kommunalen Spitzenverbänden. Für die Reform des Mehrwertsteuersystems ist zwar eine Kommission gegründet worden, sie hat jedoch kein einziges Mal getagt. Dabei standen die Chancen gut, mit einer Vereinheitlichung der Mehrwertsteuer das Wahlversprechen einer Steuervereinfachung zu erfüllen. Auch in den folgenden Legislaturperioden ist es versäumt worden, ein Steuersystem zu schaffen, das den Gerechtigkeitsvorstellungen vieler Bürgerinnen und Bürger entspricht.

Die in den vergangenen Jahrzehnten von der Politik angekündigten großen Reformen des deutschen Steuerrechts haben sich als Trugbilder herausgestellt. Immer wieder hieß es, das Ziel sei ein gerechtes und einfaches Steuersystem:

1. Die „Organische Steuerreform“ von 1954/55, die eine grundlegende Neuorientierung des Steuersystems bewirken sollte, wurde nicht durchgeführt. 
2. In der Regierungserklärung vom 28. Oktober 1969 wurde verkündet, ein gerechtes, einfaches und überschaubares Steuersystem zu schaffen.

3. Mit der Steuerreform 1990 wurde hervorgehoben: „Ziel ist ein gerechtes und einfacheres Steuersystem".

4. Im Koalitionsvertrag vom 26. Oktober 2009 wurde herausgestellt: „Wir werden das Steuerrecht spürbar vereinfachen und von unnötiger Bürokratie befreien.“

Im Ergebnis muss festgehalten werden: Das heutige Steuerrecht ist durch eine Fülle von Bevorzugungs- und damit zwangsläufig auch von Benachteiligungssachverhalten gekennzeichnet; es ist undurchschaubar, verwirrend und widersprüchlich.

Der Gesetzgeber und die Finanzverwaltung haben das Steuersystem zugunsten des Fiskus immer weiter vervollständigt und durch Nichtanwendungserlasse von finanzgerichtlichen Urteilen und durch Jahressteuergesetze die Rechtsprechung der Finanzgerichte unterlaufen. Die sich daraus ergebenden Lücken im System werden von den rechtsberatenden Berufen zugunsten ihrer Mandantschaft genutzt. Die Folge sind immer schnellere Regeländerungen durch den Gesetzgeber, die keine Planungssicherheit mehr gewährleisten, als ungerecht empfunden werden und jeden Leistungsgedanken im Keim ersticken. So ist es vor diesem Hintergrund nicht verwunderlich, wenn alle legalen Möglichkeiten von den Steuerpflichtigen ausgeschöpft werden.

Klaus Vogel hat die Situation wie folgt gekennzeichnet:

Gewiß ist das Steuerrecht schon seit langem, spätestens aber seit dem Ersten Weltkrieg, ein kompliziertes, für den Laien schwer überschaubares Rechtsgebiet. Heute kann aber auch ein Fachmann dieses Gebiet kaum noch überblicken [...]. Schon seit den siebziger und achtziger Jahren mehren sich deshalb die Äußerungen von hohen Richtern, Anwälten und Wissenschaftlern, die das geltende Steuerrecht als „Chaos“, „Dschungel“ oder gar „Perversion“ bezeichnen. In diesem Chaos gelingt es den Hochverdienenden, die sich eine teure Steuerberatung leisten können, ihre Steuerpflichten legal zu vermindern. Die Geringverdienenden haben diese Möglichkeit nicht; sie zahlen, wie dies ein namhafter Betriebswirt ausgedrückt hat, eine „Dummensteuer“. ${ }^{2005}$

Diesen Begriff der „Dummensteuer“ hat Gerd Rose geprägt, der darunter Steuerlasten versteht, „die nicht entstanden wären, wenn der Steuerpflichtige das gleiche wirtschaftliche Ziel unter klugem Einsatz der vorhandenen Gestaltungsmöglichkeiten anders erreicht hätte. ${ }^{{ }^{1206}}$ Mit dieser Definition trägt Rose dem Tatbestand Rech-

1205 Vogel, Klaus: Verfassungsrechtsprechung zum Steuerrecht, S. 9 f.

1206 Rose, Gerd: Über die Entstehung von „Dummensteuern“ und ihre Vermeidung, in: Die Steuerrechtsordnung in der Diskussion. Festschrift für Klaus Tipke zum 70. Geburtstag, Köln 1995, S. 153. 
nung, dass Laien nicht in der Lage sind, die Lücken und Privilegien der hochgradig widersprüchlichen, inkonsistenten und intransparenten Steuergesetze zu nutzen. ${ }^{1207}$

An der beispielhaft untersuchten Unternehmensbesteuerung zeigt sich, dass Steuerpflichtige, die ihren finanziellen Nutzen unter Berücksichtigung von Steuern maximieren wollen, in der Vergangenheit den verändernden Belastungsunterschieden in der Unternehmensbesteuerung durch Steuergestaltung ausgewichen sind. Der Gesetzgeber war deshalb wiederholt gezwungen zu handeln, um das Ausmaß der rechtsformabhängigen Belastungsunterschiede zu vermindern bzw. diese zu unterbinden.

Die Verletzung fundamentaler Besteuerungsprinzipien durch den Gesetzgeber hat zu einem großen Bedürfnis nach einer Fundamentalreform der Ertragsteuern geführt. Ziel einer solchen Reform wäre es, die lenkungs- und verteilungspolitischen Maßnahmen aus dem Steuerrecht herauszunehmen und sich auf eine Ordnung des Steuerrechts zu besinnen, welche die Steuerlasten gleichmäßig allen Bürgerinnen und Bürgern nach Maßgabe ihrer wirtschaftlichen Leistungsfähigkeit zuweist. ${ }^{1208}$

Unabhängig davon bestimmen der Zustand und die Rechtsanwendung des deutschen Steuerrechts wesentlich die Steuermoral der Bundesbürger. Es ist daher dringend geboten, ein grundlegend erneuertes, zukunftsfähiges deutsches Steuersystem zu schaffen, das einfacher, sozialer, transparenter und damit wieder gerechter sowie zugleich international wettbewerbsfähig wäre. ${ }^{1209}$ Aus Sicht der Kommission „Steuergesetzbuch“ sind sechs Grundvoraussetzungen für den Erfolg eines erneuerten deutschen Steuersystems zu beachten:

1. Es muss für die Bürger verständlich sein, als sozial ausgewogen akzeptiert werden und den Menschen so wenig Zeitaufwand wie möglich abverlangen.

2. Es muss international wettbewerbsfähig sein und einen Schritt zur Harmonisierung der Grundlagen der Ertragsbesteuerung in Europa vorbereiten.

3. Es muss die Wettbewerbssituation deutscher und in Deutschland tätiger Unternehmen deutlich verbessern und sie nachhaltig und praxistauglich entlasten, insbesondere von Bürokratie.

4. Es muss die kommunale Autonomie stärken, indem es Städten und Gemeinden eine breitere Einnahmenbasis und ein stetiges Aufkommen verschafft.

5. Es darf allenfalls in der Startphase Bund und Ländern eine maßvolle Anschubfinanzierung abverlangen, muss mittel- und langfristig indes kräftige Wachstumsimpulse setzen und damit zu mehr Steuereinnahmen und weniger Sozialausgaben beitragen.

1207 Beck, Hanno/Prinz, Aloys: Zahlungsbefehl von Mord-Steuern, Karussell-Geschäften und Millionärs-Oasen, München 2010, S.2.

1208 Eilfort, Michael/Lang, Joachim: Steuerpolitisches Programm, S. 44.

1209 Ebd., S. 52. 
6. Es muss schließlich für alle Beteiligten nachvollziehbar und berechenbar sein, Rechts- und Planungssicherheit gewährleisten und damit Vertrauen für Investition und Konsum schaffen. ${ }^{1210}$

Der Rückblick auf die 400-jährige Ideengeschichte der Steuergerechtigkeit sollte bewusst machen, dass in einer sich schnell ändernden Welt Lehren aus den Erfahrungen der Vergangenheit gezogen werden müssen, um eine gerechte Steuerpolitik in Gegenwart und Zukunft zu gestalten.

Dieses Buch wird unter der Creative Commons Namensnennung 4.0 International Lizenz (http://creativecommons.org/licenses/by/4.0/deed.de) veröffentlicht, welche die Nutzung, Vervielfältigung, Bearbeitung, Verbreitung und Wiedergabe in jeglichem Medium und Format erlaubt, sofern Sie den/die ursprünglichen Autor(en) und die Quelle ordnungsgemäß nennen, einen Link zur Creative Commons Lizenz beifügen und angeben, ob Änderungen vorgenommen wurden.

Die in diesem Buch enthaltenen Bilder und sonstiges Drittmaterial unterliegen ebenfalls der genannten Creative Commons Lizenz, sofern sich aus der Abbildungslegende nichts anderes ergibt. Sofern das betreffende Material nicht unter der genannten Creative Commons Lizenz steht und die betreffende Handlung nicht nach gesetzlichen Vorschriften erlaubt ist, ist für die oben aufgeführten Weiterverwendungen des Materials die Einwilligung des jeweiligen Rechteinhabers einzuholen. 